\title{
Genetically encoded fluorescent sensors reveal dynamic regulation of NADPH metabolism
}

\author{
Rongkun Tao ${ }^{1,9}$, Yuzheng Zhao ${ }^{1,3,4,9}$, Huanyu Chu $^{2,9}$, Aoxue Wang ${ }^{1}$, Jiahuan Zhu ${ }^{1}$, Xianjun \\ Chen ${ }^{1,3,4}$, Yejun Zou ${ }^{1}$, Mei Shi ${ }^{1}$, Renmei Liu ${ }^{1}$, Ni Su ${ }^{1}$, Jiulin Du ${ }^{3}$, Hai-Meng Zhou ${ }^{5}$, Linyong \\ Zhu $^{6}$, Xuhong Qian ${ }^{7}$, Haiyan Liu ${ }^{2}$, Joseph Loscalzo ${ }^{8}$, and Yi Yang ${ }^{1,3}$ \\ ${ }^{1}$ Synthetic Biology and Biotechnology Laboratory, State Key Laboratory of Bioreactor \\ Engineering, Shanghai Collaborative Innovation Center for Biomanufacturing Technology, East \\ China University of Science and Technology, 130 Mei Long Road, Shanghai 200237, China \\ ${ }^{2}$ School of Life Sciences, Hefei National Laboratory for Physical Sciences at the Microscale, \\ University of Science and Technology of China, Hefei, Anhui 230026, China \\ ${ }^{3}$ State Key Laboratory of Neuroscience, CAS Center for Excellence in Brain Science, Institute of \\ Neuroscience, Chinese Academy of Sciences, Shanghai 200031, China \\ 4Shanghai Key Laboratory of New Drug Design, School of Pharmacy, East China University of \\ Science and Technology, 130 Mei Long Road, Shanghai 200237, China \\ ${ }^{5}$ Zhejiang Provincial Key Laboratory of Applied Enzymology, Yangtze Delta Region Institute of \\ Tsinghua University, Jiaxing 314006, China \\ ${ }^{6}$ School of Chemistry and Molecular Engineering, East China University of Science and \\ Technology, 130 Mei Long Road, Shanghai 200237, China \\ ${ }^{7}$ Shanghai Key Lab. of Chemical Biology, School of Pharmacy, East China University of Science \\ and Technology, Shanghai 200237, China \\ ${ }^{8}$ Department of Medicine, Brigham and Women's Hospital, Harvard Medical School, Boston, MA \\ 02115, USA
}

\section{SUMMARY}

Reduced nicotinamide adenine dinucleotide phosphate (NADPH) is essential for for biosynthetic reactions and antioxidant functions; however, detection of NADPH metabolism in living cells remains technically challenging. We develop and characterize ratiometric, $\mathrm{pH}$-resistant, genetically encoded fluorescent indicators for NADPH (iNap sensors) with various affinities and wide

\footnotetext{
Correspondence should be addressed to H.L (hyliu@ustc.edu.cn) and Y.Y. yiyang @ecust.edu.cn).

${ }^{9}$ These authors contributed equally to this work

Note: Any Supplementary Information and Source Data files are available in the online version of the paper.

AUTHOR CONTRIBUTIONS

Y. Y., H.L., H.C. and R.T. designed and validated the sensors in vitro. Y. Y., R.T. and Y. Zhao designed the live cell imaging, metabolic assays and signaling study experiments. R.T., Y. Zhao, A.W., J.Z., X.C., Y. Zou, M.S., R.L. and N.S. performed experiments. J.D.,

H.Z., L.Z., X.Q., and J.L. gave technical support and conceptual advice. Y.Y., H.L., Y. Zhao, R.T., H.C. and J.L. analyzed the data and wrote the manuscript.
}

COMPETING FINANCIAL INTERESTS

The authors declare no competing financial interests. 
dynamic range. The iNap sensors permitted quantification of cytosolic and mitochondrial NADPH pools that were controlled by cytosolic $\mathrm{NAD}^{+}$kinase levels, and revealed cellular NADPH dynamics under oxidative stress depending on glucose availability. We find that mammalian cells have a strong tendency to maintain physiological NADPH homeostasis, which is regulated by glucose-6-phosphate dehydrogenase (G6PD) and AMP kinase (AMPK). Moreover, using the iNap sensors we monitor NADPH fluctuations during the activation of macrophage cells or wound response in vivo. These data demonstrate that the iNap sensors will be valuable tools for monitoring NADPH dynamics in live-cells, and gaining new insights into cell metabolism.

\section{INTRODUCTION}

NADPH is a ubiquitous cofactor in all living organisms that supports reducing capacity, macromolecular biosynthesis, superoxide and nitric oxide production, and detoxification of drugs and xenobiotics ${ }^{1-5}$. Perturbed NADPH metabolism leads to impaired cell functions, thereby increasing the risks of numerous disease-associated pathologies, such as inflammation, aging, cancer, diabetes mellitus, and neurodegeneration ${ }^{2,5-7}$. Most of the NADPH regenerating and consuming enzymes are highly compartmentalized, thus, spatiotemporal tracking of NADPH metabolism in living cells is crucial for understanding the role of this cofactor in cell biology.

Existing methods for NADPH measurement, such as chromatography, mass spectrometry and enzymatic cycling assays are invasive and may allow sample oxidation during processing. Fluorescence lifetime imaging was shown to distinguish NADPH and NADH fluorescence in live cells ${ }^{8}$; however, it requires technically specialized instrumentation and analysis, which may not be readily accessible. Genetically encoded fluorescent sensors capable of live cell monitoring were developed for pyridine nucleotides such as $\mathrm{NADH}^{9-12}$, $\mathrm{NAD}^{+13}$, and $\mathrm{NADP}^{+14}$. Among them, SoNar is a highly responsive sensor for the $\mathrm{NADH} / \mathrm{NAD}^{+}$ratio with superior properties useful for live cell and in vivo studies ${ }^{10,15,16}$. We designed the iNap NADPH sensors by engineering SoNar's binding site to switch ligand selectivity from NADH to NADPH.

\section{RESULTS}

\section{Structure-guided engineering of NADPH sensors}

SoNar is a chimera of circularly permuted YFP (cpYFP) and the NAD(H) binding domain of Rex from Thermus aquaticus (T-Rex) ${ }^{10}$; the T-Rex ligand-binding pocket is illustrated in Fig. 1a. We collected sets of NAD(H)-binding and NADP(H)-binding proteins from the Protein Data Bank (Supplementary Table 1), and compared their binding pockets using Comparison of Protein Active Site Structures (CPASS) ${ }^{17}$. In NADP(H)-binding proteins, positively charged residues are enriched close to the binding pockets of 2'-phosphate in NADP $(H)$ (Supplementary Fig. 1a), favoring electrostatic interactions. By contrast, negatively charged residues are enriched in the same location of $\mathrm{NAD}(\mathrm{H})$-binding proteins. A structural comparison of T-Rex and an NADP $(\mathrm{H})$ binder $^{18}$ showed that the 2 '-phosphate group of $\mathrm{NADP}(\mathrm{H})$ would sterically clash with the protein loop in T-Rex, which has limited conformational flexibility due to Pro115 (Fig. 1a, Supplementary Fig. 1b). Furthermore, 
NADP $(H)$-binding proteins tend to contain a larger portion of polar residues in their adenine binding pocket (Supplementary Fig. 1c and Supplementary Table 2). To switch T-Rex from a NADH binder into a NADPH binder, we therefore designed mutations that switched the charges, reduced the rigidity of the loop around the 2'-hydroxyl functionality of the ligand, and/or increased the polarity of the adenine binding pocket (Supplementary Table 3).

\section{In vitro characterization of iNap sensors}

We tested mutants of SoNar with combinations of the designed point mutations, which were all fluorescent when expressed in E.coli and strongly responded to NADPH, but not to other adenine dinucleotides (Fig. 1b and Supplementary Fig. 2a). These NADPH reporters had various affinities, with apparent dissociation constants $\left(K_{\mathrm{d}}\right)$ ranging from $\sim 1.3 \mu \mathrm{M}$ to $\sim 29$ $\mu \mathrm{M}$ as measured by the ratio of fluorescence excited at $420 \mathrm{~nm}$ and $485 \mathrm{~nm}\left(\mathrm{R}_{420 / 485}\right)$ (Supplementary Table 3). Considering the maximum fluorescence ratio change, affinity, and expression level, we chose mutants with apparent $\mathrm{K}_{\mathrm{d}} \mathrm{S}$ of $\sim 2.0 \mu \mathrm{M}, \sim 6.0 \mu \mathrm{M}, \sim 25 \mu \mathrm{M}$, and $\sim 120 \mu \mathrm{M}$ for further characterization and denoted these NADPH sensors iNap1-iNap4 (Supplementary Table 3 and Supplementary Fig. 2b).

iNap1 has two excitation peaks around $420 \mathrm{~nm}$ and $500 \mathrm{~nm}$, and one emission peak near 515 nm (Fig. 1c and Supplementary Fig. 2c,d). Upon NADPH binding, iNap1 exhibited a 3.5fold increase and 2.5-fold decrease in fluorescence excited at $420 \mathrm{~nm}$ and $485 \mathrm{~nm}$, respectively (Fig. 1d), leading to a $900 \%$ ratiometric fluorescence change that was essentially unaffected by temperature fluctuation between $20^{\circ} \mathrm{C}$ and $42{ }^{\circ} \mathrm{C}$ (Fig. 1e, Supplementary Fig. 2e). iNap1 sensor possesses high selectivity toward NADPH, showing no apparent fluorescence changes toward related nucleotides (Fig. 1e and Supplementary Fig. 2f-h). iNap1 fluorescence excited at $420 \mathrm{~nm}$, which represented the pronated chromophore $^{19}$, is insensitive to $\mathrm{pH}$ (Fig. 1f). Fusion of iNap1 and the red fluorescent protein mCherry allows ratiometric and pH-resistant measurement (Supplementary Fig. 3a). The iNap1's fluorescence excited at $485 \mathrm{~nm}$ depends on $\mathrm{pH}$; however, its $\mathrm{K}_{\mathrm{d}}$ is rather resistant to $\mathrm{pH}$ changes (Supplementary Fig. 3b-e). We created a control iNap sensor, termed iNapc, by introducing four mutations in T-Rex, which completely abolished ligand binding (Supplementary Table 3). iNapc and iNap1, either in the absence or in the presence of NADPH, displayed similar sensitivities to $\mathrm{pH}$. When modest $\mathrm{pH}$ fluctuations occurred, the $\mathrm{pH}$ effects on iNap1 fluorescence excited at $485 \mathrm{~nm}$ could be accounted for by normalizing to iNapc (Supplementary Fig. 3f-h). The fluorescence of iNap1 responded rapidly to sequential addition of NADPH and redox cycling agents ${ }^{10}$ (Fig. 1g), supporting its use in real-time measurements. Fluorescence properties and responses of other iNaps are very similar if not identical to that of iNap1, but with different affinities (Fig. 1h), suggesting the few mutations that they carried, which are distantly located from the cpYFP domain, mainly affect the selective binding of the adenine moiety of $\mathrm{NAD}(\mathrm{P}) \mathrm{H}$, but not the fluorescence response per se. Taken together, iNap sensors manifest excellent sensitivity and selectivity, and a large dynamic range.

\section{Subcellular distribution and regulation of NADPH in mammalian cells}

iNap1 showed intense uniform fluorescence when stably expressed in HeLa cells (Fig. 2a, Supplementary Fig. $4 a-c)$. The images of fluorescence ratio $\left(\mathrm{R}_{407 / 482}\right)$ showed no marked 
differences between cells (Supplementary Fig. 4d-f), or between cytosolic and nuclear signals (Fig. 2a), suggesting that these cells had similar NADPH level, and that NADPH was readily exchangeable between the cytoplasm and nucleus. iNap sensors can be selectively targeted to the mitochondrial matrix using a targeting sequence (Fig. 2b). Free NADPH level in the cytosol and mitochondrial matrix were determined to be $3.1 \pm 0.3 \mu \mathrm{M}$ and $\sim 37 \pm 2 \mu \mathrm{M}$ using iNap1 and iNap3 (Fig. 2c,d), respectively, according to their apparent occupancies (Supplementary Fig. 5a,b). Similar estimation of free NADPH level were obtained using other iNap variants expressed in cytosol or mitochondria, consistent with their in-vitro determined $\mathrm{K}_{\mathrm{d}}$ 's (Supplementary Table 4). These sensors do not appear to substantially perturb the cellular NADPH level, which is not surprising as NADPH is in rapid equilibrium with millimolar concentration of various metabolites maintained by malic enzyme or isocitrate dehydrogenase $\mathrm{e}^{20,21}$.

Human cells have two compartment-specific NADKs, i.e., cytosolic NADK and mitochondrial NADK2, which catalyzed the synthesis of $\mathrm{NADP}^{+5,22}$. Overexpression or knockdown of NADK resulted in a $\sim 5$-fold increase and $\sim 6$-fold decrease in cytosolic NADPH, respectively (Fig. 2a, c and Supplementary Fig. 5c,d). Intriguingly, mitochondrial NADPH was also markedly affected by cytosolic NADK levels as showed by iNap3 fluorescence (Fig. 2b, d and Supplementary Fig. 5b), the pH-insensitive measurement by mCherry-iNap3, and the data from enzymatic cycling assays (Supplementary Fig. 5e-h). These data demonstrate the key role of NADK for the maintenance of both cytosolic and mitochondrial pool of NADPH.

To measure NADPH and NADH levels in HeLa cells simultaneously, we expressed iNap1 in the cytosol using a nucleus-excluded signal peptide, together with SoNar in the nucleus with a nuclear localization signal (Fig. 2e). In cells treated with lactate dehydrogenase (LDH) inhibitor oxamate which decreased the cytosolic $\mathrm{NAD}^{+} / \mathrm{NADH}$ ratio ${ }^{10}$, a marked change in SoNar fluorescence but not iNap1 fluorescence or iNapc fluorescence was observed (Fig. 2e). In cells treated with a glycolysis inhibitor, 2-deoxy-glucose (2-DG), a marked change occurred in the fluorescence of the ATP:ADP ratio biosensor PercevalHR which was consistent with previous study ${ }^{23}$, but not of iNap1 (Supplementary Fig. 5i). These data suggested that iNap specifically reports NADPH levels in living cells, without interference from other relevant adenine nucleotides. In resting HeLa cells, knockdown of the rate limiting enzyme G6PD of pentose phosphate pathway (PPP), the main contributor of cytosolic NADPH regeneration, slightly decreased cytosolic NADPH levels; whereas knockdown of the 6-phosphogluconate dehydrogenase (PGD) in the same pathway showed little effect (Supplementary Fig. 5j, k).

We next studied NADPH level in macrophages by flow cytometry. As illustrated by both the decrease and the wider distribution of iNap1 fluorescence ratio $\left(\mathrm{R}_{405 / 488}\right)$, lipopolysaccharide (LPS) and IFN- $\gamma$ treatment induced non-homogeneously decrease of NADPH level in RAW264.7 mouse macrophages (Fig. 2f and Supplementary Fig. 51). The $\mathrm{R}_{405 / 488}$ of iNapc slightly increased in these activated macrophages, which may be explained by the acidification of the cells (Fig. $2 \mathrm{~g}$ and Supplementary Fig. 5l). Similar results were obtained when iNap1 or iNapc fluorescence was measured by a microplate reader (Supplementary Fig. 5m). These results are consistent with previous report that macrophages 
synthesize nitric oxide and consume NADPH upon activation ${ }^{24}$, and suggest iNap sensor may be useful to differentiate activated macrophages from resting macrophages.

\section{NADPH dynamics of glucose-deprived cells in the setting of oxidative stress}

NADPH provides the reducing power for both the glutathione and thioredoxin systems to combat oxidative stress. In glucose-starved HeLa cells, cellular glucose and NADH levels significantly decreased, whereas the NADPH level and thiol redox state remained unchanged, as reported by genetically encoded sensors FLII ${ }^{12} \mathrm{Pglu}-700 \mu^{25}$ for glucose, SoNar, iNap1, and roGFP1 ${ }^{26}$ for protein thiol oxidation, respectively (Supplementary Fig. 6a). When these cells were challenged with diamide, a general oxidant, the ratio of iNap1 fluorescence decreased immediately and then gradually recovered (Fig. 3a-c). Such a recovery may reflected the enhanced NADPH production under oxidative stress, as G6PD is allosterically stimulated by the elevated level of $\mathrm{NADP}^{+}$resulting from oxidation of $\mathrm{NADPH}^{27,28}$. The ratio of roGFP1 fluorescence increased and recovered with kinetics more slowly comparing to that of iNap1 under low diamide concentrations (Fig. 3a-d), implying that fluctuation of cytosolic NADPH occurred before that of thiol redox state. Repeated addition of diamide led to similar fluorescence changes in iNap1-expressing or roGFP1positive cells, confirming that these sensors exhibited excellent reversibility and sensitivity in living cells (Fig. 3e). As a control, minimal changes of fluorescence were observed in iNapc-expressing cells with diamide treatment (Fig. 3a, b), thereby excluding the possibilities of interference of fluorescence emission due to $\mathrm{pH}$ variations or redox state of the cpYFP domain. Similar results were also seen in $\mathrm{H}_{2} \mathrm{O}_{2}$-treated $\mathrm{HeLa}$ cells (Supplementary Fig. 6b). Dynamic changes of NADPH level upon diamide treatment were inhibited by the glutathione reductase and thioredoxin reductase inhibitor, carmustine (BCNU), which blocked these NADPH consuming and thiol regeneration pathways (Supplementary Fig. 6c). Furthermore, the recovery of NADPH and thiol oxidation in diamide-treated HeLa cells was almost completely inhibited by the knockdown of G6PD or treatment with a pharmacologic G6PD inhibitor, DHEA (Fig. 3f, g and Supplementary Fig. 6d). Knockdown of PGD, another NADPH-generating enzyme in the PPP, showed no apparent difference with control (Supplementary Fig. 6e). These data suggested that NADPH recovery under oxidative stress was mainly regulated by G6PD in glucose-deprived cells.

\section{NADPH dynamics of glucose-fed cells in the setting of oxidative stress}

In glucose-fed cells, addition of diamide or $\mathrm{H}_{2} \mathrm{O}_{2}$ dose-dependently and mildly decreased the ratio of iNap1 fluorescence (Fig. 4a, b and Supplementary Fig. 7a, b). In control, roGFP1 responses to diamide or $\mathrm{H}_{2} \mathrm{O}_{2}$ were similar for glucose-fed cells and glucose-starved cells (Fig. $4 \mathrm{c}$ and Supplementary Fig. 7b), suggesting that an efficient anti-oxidative stress response was induced independent of glucose levels. BCNU only partially reversed the diamide-induced decrease of cytosolic NADPH in glucose-fed cells (Supplementary Fig. $7 c)$.

The iNap1 response to diamide was strictly dependent on glucose but was independent of other metabolites added into the medium, such as glutamine, pyruvate (Fig. $4 \mathrm{~d}$ and Supplementary Fig. 7d). The glucose concentration for the half-maximal iNap1 and SoNar 
fluorescence response is $\sim 0.16 \mathrm{mM}$ and $\sim 0.5 \mathrm{mM}$, respectively, in HeLa cells

(Supplementary Fig. 7e), suggesting different glucose sensitivities of the PPP and glycolysis. In the presence of glucose, NADPH level largely recovered after initial decrease under oxidative stress in DHEA treated cells, but not in G6PD knockdown cells (Fig. 4e,f).

\section{AMPK regulates NADPH homeostasis in glucose-fed cells under oxidative stress}

The AMPK system senses nutrient and energy and is activated by energy stress and oxidative stress ${ }^{29}$, and helps to maintain NADPH homeostasis by decreasing NADPH consumption in reductive synthesis ${ }^{30}$. AMPK activation as shown by phosphorylated AMPK (pAMPK) and phosphorylated acetyl-CoA carboxylase (pACC) is moderate in glucosedeprived cells, rare in glucose-fed cells with or without G6PD-knockdown, but surprisingly abundant in DHEA-treated glucose-fed cells (Fig. 5a). In HeLa cells, AMPK kinase LKB1 is deficient ${ }^{31}$, thus, AMPK activation is mainly mediated by $\mathrm{Ca}^{2+} /$ calmodulin-dependent protein kinase kinase $(\mathrm{CaMKK})^{29}$. We therefore treated glucose-fed HeLa cells with the $\mathrm{Ca}^{2+}$ ionophore ionomycin to increase AMPK activity ${ }^{31}$ or with dorsomorphin to block AMPK activity (Fig. 5b). Diamide-induced NADPH oxidation and cell death were significantly attenuated by ionomycin (Fig. 5c, d, Supplementary Fig. 8a), but partially enhanced by dorsomorphin (Fig. 5c, d, Supplementary Fig. 8a). The protective effect of ionomycin on diamide-induced cell injury was significantly weakened by dorsomorphin (Fig. 5d), consistent with the results of parallel Western Blot analysis of pAMPK and pACC (Fig. 5b). These data highlight that $\mathrm{Ca}^{2+}$-mediated AMPK activation in HeLa cells is a prosurvival response under oxidative stress that acts via NADPH maintenance.

Interestingly, DHEA-treated cells are much more resistant to oxidative stress than G6PDknockdown cells, suggesting that it may have roles independent of G6PD inhibition (Fig. 5e). Further studies showed that the effects of DHEA on NADPH recovery and cell viability under oxidative stress were suppressed by AMPK inhibition (Fig. 5b, e, f). These data suggested that DHEA possessed dual biological functions: AMPK activation and G6PD inhibition, with opposing effects on cell survival. In fact, DHEA enhanced cell viability under oxidative stress in glucose-fed cells (Fig. 5e), suggesting that its negative effect on G6PD inhibition and NADPH regeneration is offset by the activation of AMPK.

Using a $\mathrm{Ca}^{2+}$ probe, Fluo-4 AM, and the PercevalHR sensor, we found that DHEA treatment in glucose-fed cells caused a robust increase of cytosolic $\mathrm{Ca}^{2+}$ level (Fig. 5g) and ADP:ATP ratio (Fig. 5h), both are known to activate the AMPK pathway ${ }^{29}$, 32. G6PD-knockdown also increased the cellular ADP:ATP ratio; however, cytosolic $\mathrm{Ca}^{2+}$ level elevation did not occur compared to control cells (Fig. 5g, h), consistent with the low level of AMPK activation in these cells (Fig. 5a). In glucose starved cells, inhibition of AMPK only mildly delayed the recovery of NADPH level under oxidative stress (Supplementary Fig. 8b). In Fig. 5i-1, we summarize PPP and AMPK regulated cytosolic NADPH flux in glucose-fed cells under oxidative stress under different conditions. A working model is shown for AMPK activation and NADPH level regulation in glucose starved cells under oxidative stress (Supplementary Fig. 8c). 


\section{Visualization of NADPH dynamics at a tissue-scale in zebrafish}

To visualize NADPH dynamics in vivo, we co-microinjected mRNAs of iNap1 and HyPerRed ${ }^{33}$, a red $\mathrm{H}_{2} \mathrm{O}_{2}$ sensor, into the one-cell-stage zebrafish zygote (Fig. 6a). Both sensors expressed well and their fluorescence was visible in the zebrafish larvae at 2 days post fertilization. Upon local injury of the tail fin, marked decrease of NADPH level occurred within 25 mins at the wound margin as showed by iNap1's fluorescence (Fig. 6b). The signal of HyPerRed also increased, which co-localized well with the site where NADPH level drops (Fig. 6b). No obvious fluorescence change of iNapc-expressing zebrafish larvae was observed in parallel experiments under the same conditions (Supplementary Fig. 9). Such decrease of NADPH level and increase of $\mathrm{H}_{2} \mathrm{O}_{2}$ level were blocked by diphenyleneiodonium (DPI), a NADPH oxidase inhibitor (Fig. 6b). It is known that in zebrafish, wounding induces rapid production of $\mathrm{H}_{2} \mathrm{O}_{2}$ through the catalysis of dual oxidase at the expense of NADPH consumption ${ }^{34}$. These results agree well with the previous reports, and demonstrate that iNap sensor can be used to measure NADPH dynamics in vivo.

\section{DISCUSSION}

For reference, we attach a diagram that explains the central nodes for cellular NADPH metabolism (Supplementary Fig. 10). Responses of iNap sensors are based on fluorescence intensity, which can be readily analyzed using fluorescent microscopes, flow cytometers or microplate readers. iNap sensors have inherited most of their favorable characteristics from SoNar ${ }^{10}$, including intense fluorescence, rapid responsiveness, $\mathrm{pH}$-insensitivity, large dynamic range, targeting to subcellular organelles, ratiometric imaging and dynamic measurements in living cells and in vivo. These characteristics make them one of the most effective genetically encoded sensors currently available.

The large dynamic range of iNap in live cells making iNap very useful for tracking subtle differences in cellular NADPH/NADP ${ }^{+}$redox state. The availability of iNap with various affinities further expands the utility of iNap sensors for applications in different cells and subcellular organelles. For mammalian cells, we suggest iNap1 for the measurement of low abundant cytosolic NADPH, and iNap3 for the measurement of higher level mitochondrial NADPH. The non-binding control sensor iNapc is useful as a control to identify fluctuation of cellular NADPH level, and to correct iNap's fluorescence when $\mathrm{pH}$ fluctuations occurs, although $\mathrm{pH}$ effects were not obvious in the other studies of cpYFP-based, $\mathrm{pH}$ sensitive sensors ${ }^{19,35,36}$. Facile $\mathrm{pH}$ correction can be carried out in quantitative studies involving microplate reading by measuring iNap's and iNapc's fluorescence in parallel samples. For the microscopy studies, it is possible to correct $\mathrm{pH}$ effects in situ by expressing iNap sensor in cytosol, and iNapc in nucleus; corrections can be made after quantification of iNap and iNapc signals for each cells based on the assumption that metabolites diffuse freely between cytosol and nucleus. For quantitative imaging under $\mathrm{pH}$ fluctuation conditions, iNap fluorescence can be measured with the $\mathrm{pH}$ resistant $420 \mathrm{~nm}$ excitation only, at the expense of reduced dynamic range and non-ratiometric mode. Ratiometric and $\mathrm{pH}$-resistant measurement may also be achieved by using the fusion of iNap with the red fluorescent protein mCherry, by measuring the green/red fluorescence ratio. mCherry-iNap sensor has additional benefit to avoid the recently identified systematic error for measuring occupancy 
of a ratiometric sensor ${ }^{37}$. Furthermore, it is possible to determine NADPH and NADP ${ }^{+}$ dynamics simultaneously when iNap is utilized jointly with the recently reported NADP ${ }^{+}$ sensors ${ }^{14}$, which are spectrally tunable, enabling them to be used for multi-color imaging.

Many cancer cells are characterized by increased aerobic glycolysis and high levels of oxidative stress ${ }^{1}$. Besides, solid tumors may have a nutrient-poor, glucose depleting microenvironment, due to impaired vasculature or rapid growth ${ }^{38}$. Our data showed that under glucose starvation, NADH and glucose levels in Hela cells rapidly decreased, however, NADPH level and thiol redox state remained constant. These data suggested that cellular glucose metabolism favors the NADPH-producing PPP rather than NADHproducing glycolysis under glucose limiting conditions, probably to maintain ideal redox and bioenergetic balance for cell survival and growth. In glucose-deprived cells treated with oxidant, NADPH decreased immediately and then gradually recovered. Such a recovery of NADPH level can be explained by the enhanced NADPH regeneration as a result of G6PD activation, and reduced NADPH consumption as a result of AMPK activation. G6PD is known to be allosterically stimulated by $\mathrm{NADP}^{+}$and strongly inhibited by $\mathrm{NADPH}^{27,28}$. AMPK is able to regulate NADPH homeostasis by decreasing NADPH consumption by reductive synthesis and increasing NADPH generation by means of fatty-acid oxidation, in order to promote tumor cell survival during energy stress ${ }^{30}$. Intriguingly, we found that the human physiological steroid hormone DHEA, also a well-known G6PD inhibitor, caused a robust increase of cytosolic $\mathrm{Ca}^{2+}$ levels, activated AMPK pathway, rerouted NADPH flux to antioxidant functions and helped to maintain NADPH homeostasis under oxidative stress. AMPK has been an attractive therapeutic target in various metabolic diseases, including type 2 diabetes, obesity, aging, and cancer ${ }^{39-42}$, thus, we should consider the potential application of DHEA or similar compounds in these settings.

Given the comparable significance of NADPH/NADP ${ }^{+}$and NADH/NAD ${ }^{+}$couples in biologically and medically important processes, we anticipate that iNap sensors together with SoNar should open the door to a more complete understanding of cellular and subcellular metabolism, and will be embraced as a useful alternative to existing methods for detecting NADPH in living cells and in vivo.

\section{ONLINE METHODS}

\section{Rational Design of NADPH Sensors}

We performed comparative analyses of $343 \mathrm{NAD}(\mathrm{H})$-binding and $211 \mathrm{NADP}(\mathrm{H})$-binding proteins (Supplementary Table 1) using the program, CPASS, which carries out structure alignments between the ligand binding sites of proteins in a sequence-order-independent manner. Residues containing at least one side chain atom within $5 \AA$ from selected ligand atoms were selected as the binding sites. Amino acid compositions of the binding pockets around the adenine moiety and around the 2' oxygen of the ligand were analyzed separately. According to the CPASS alignments, we further isolated 16 proteins [14 NAD $(H)$-binding and 2 NADP(H)-binding] from those in Supplementary Table 1 based on their highly similar geometrical arrangements of binding residues in the $\mathrm{NAD}(\mathrm{H})$ binding protein, $1 \mathrm{xcb}$, and inspected their binding residue substitutions as compared with 1xcb. 


\section{Materials}

PrimeSTAR HS DNA Polymerase was purchased from TaKaRa; Restriction endonucleases and T4 DNA ligase were obtained from Fermentas; and the nucleotides NADPH, NADH, $\mathrm{NAD}^{+}$, and $\mathrm{NADP}^{+}$were purchased from Roche and prepared just before use, ATP, ADP, AMP, diamide, glucose, oxamate, dehydroepiandrosterone, carmustine, dorsomorphin, malate, isocitrate, 2-deoxy-d-glucose, MTT, glucose-6-phosphate, 6-phosphogluconate, phenazine methosulfate, yeast G6PD, NAM, thiazolylblue, phenazine ethosulfate and lipopolysaccharides (LPS) were all obtained from Sigma-Aldrich. Glutamine and pyruvate were provided from Invitrogen. Digitonin and $\mathrm{H}_{2} \mathrm{O}_{2}$ were obtained from Calbiochem (Merck, Germany), and KP372-1 was purchased from Echelon Biosciences, Inc.

Recombinant murine interferon- $\gamma$ (IFN- $\gamma$ ) was obtained from Absin. Other reagents were of analytical grade and obtained from local suppliers.

\section{Plasmid Construction}

The SoNar gene was digested from the pRSET-B vector and subcloned into the pSulA-TorA vector using BamHI and HindIII. All the mutated plasmids were generated through inverse PCR by using PrimeSTAR. The pSulA-TorA plasmid harboring a mutated SulA promoter that could not be recognized by LexA ${ }^{43}$ can be used to constitutively express the recombinant protein and partially secrete it in the periplasmic space, as used for GECO probes $^{44}$. For the construction of mCherry-iNap1, we used a 20-aa linker, (GGSGG) 4 , to connect mCherry to the $\mathrm{N}$-terminal of iNap1 sensor.

To construct a mammalian cell expression vector, the entire coding sequences of iNap1, iNap3, and iNapc were subcloned into the pcDNA3.1/hygro(+) vector. The iNap3-Mit plasmid was constructed by fusing a duplicated mitochondrial targeting sequence from cytochrome c oxidase subunit VIII at the N-terminus. For nuclear and nuclear-excluded targeting, the three-fold nuclear localization sequence, (DPKKKRKV) $)_{3}$, and tandem fused MAPKK signal peptide (MALQKKLEELELDEQQRKRLEDL) 2 were inserted at the Cterminus and $\mathrm{N}$-terminus of sensors, respectively.

The NADK cDNA, PercevalHR, and FLII ${ }^{12}$ Pglu700 $\mu$ probes were obtained from Addgene. roGFP1 was a kind gift from S. James Remington (University of California San Diego). For construction of lentivirus vectors, NADK cDNA, roGFP1, and FLII ${ }^{12}$ Pglu700 $\mu$ were ligated into the pLVX-IRES-Puro plasmid. All shRNA were synthetized from GeneChem (Shanghai, China) and ligated into the pCS2 plasmid containing the puromycin-resistant gene and the red fluorescent protein, mCherry. The sequence of all shRNAs against the target genes are as follows: NADK shRNA \#1: GATGAGAGATGCCAGCCTACT, \#2: GAGCGTCCTTGTCATCAAGAA, G6PD shRNA \#1: CAACAGATACAAGAACGTGAA, \#2: GTCGTCCTCTATGTGGAGAAT, PGD shRNA (lot: GIEL1121026471)

\section{Protein Expression and Purification}

E. coli MachI cells carrying the pSulA-TorA-iNap expression plasmid were grown in $100 \mathrm{ml}$ LB media containing $100 \mu \mathrm{g} / \mathrm{ml}$ ampicillin at $30{ }^{\circ} \mathrm{C}$ for $40 \mathrm{hrs}$. Bacteria were harvested by centrifugation at $4,000 \mathrm{rpm}$ for $20 \mathrm{~min}$ at $4{ }^{\circ} \mathrm{C}$. The cell pellets were suspended in Buffer A (30 mM sodium phosphate, $500 \mathrm{mM}$ sodium chloride, and $10 \mathrm{mM}$ imidazole, $\mathrm{pH}$ 7.4), and 
lysed via sonication. Proteins were purified using a NTA column (GE Healthcare). After washing with 2 column volumes of wash buffer containing $50 \mathrm{mM}$ imidazole, the proteins were eluted from the resin using Buffer B ( $30 \mathrm{mM}$ sodium phosphate, $500 \mathrm{mM}$ sodium chloride, and $300 \mathrm{mM}$ imidazole, $\mathrm{pH}$ 7.4). The protein preparations were then desalted and exchanged into $100 \mathrm{mM}$ HEPES buffer containing $100 \mathrm{mM} \mathrm{KCl}(\mathrm{pH} 7.3)$ for in vitro characterization.

\section{In Vitro Characterization of iNap1}

The purified proteins were stored at $-80{ }^{\circ} \mathrm{C}$ until use. Measurement of the excitation and emission spectra of recombinant fluorescent sensor proteins was carried out as previously described ${ }^{9,10}$. The purified sensor protein was placed in a cuvette containing $100 \mathrm{mM}$ HEPES buffer with $100 \mathrm{mM}$ potassium chloride, $\mathrm{pH}$ 7.3. Fluorescence was measured using a Cary Eclipse spectrofluorimeter (Varian). For nucleotide titration, the protein was diluted in HEPES buffer ( $\mathrm{pH} 7.3$ ) to a final concentration of $0.2 \mu \mathrm{M}$. The fluorescence intensity of iNap1 in the absence of nucleotides was measured by using a filter-based Synergy 2 MultiMode microplate reader with 420 BP $10 \mathrm{~nm}$, or 485 BP $20 \mathrm{~nm}$, excitation and 528 BP 20 $\mathrm{nm}$, emission band-pass filters (BioTek). The stock solution of nucleotides was also prepared in HEPES buffer (pH 7.3). Each assay was performed with $50 \mu \mathrm{l}$ nucleotides and $50 \mu \mathrm{l}$ protein in black 96-well flat-bottomed plate. Fluorescence intensity was read immediately after addition of nucleotide. $\mathrm{pH}$ correction is determined by dividing the fluorescence ratio $\left(\mathrm{R}_{420 / 485}\right)$ of iNap1 by that of iNapc.

\section{Cell Culture, Transfections, and Generation of Stable Cell Lines}

HeLa cells (derived from Cell Bank of Chinese Academy of Science) and HEK293T cells (derived from Invitrogen) were grown in high glucose DMEM (Hyclone) with 10\% FBS (Gibco). All cell lines were cultured at $37{ }^{\circ} \mathrm{C}$ in a humidified atmosphere of $95 \%$ air and $5 \%$ $\mathrm{CO}_{2}$. All cell lines were tested for mycoplasma contamination and experiments were conducted under mycoplasma- negative conditions.

For transient transfection, approximately 60,000 or 20,000 HeLa cells were plated on a 4well glass bottomed dish $\left(35 \mathrm{~mm}^{2}\right)$ or 96-well plate per well (WHB), respectively. The plasmids were transfected with lipofectamine 2000 (Invitrogen) or Hieff Trans (YEASEN, China) according to the manufacturer's protocol.

The pLVX lentiviral plasmids encoding sensors (iNap, roGFP1, FLII ${ }^{12}$ Pglu700 $\mu$ ), NADK were constructed. Lentivirus was produced by co-transfecting two lentiviral packaging vectors (pMD2G and psPAX2) in HEK293T cells. Lentiviral supernatants were collected 48 and $72 \mathrm{hr}$ after transfection. HeLa cells in 6-well tissue culture plates were infected in media containing $4 \mu \mathrm{g} / \mathrm{ml}$ polybrene and centrifuged at 1,800 rpm for $1 \mathrm{hr}$. Post-infection, virus was removed and cells were selected with $0.2-1 \mu \mathrm{g} / \mathrm{ml}$ puromycin for 1 week. After 1 week, the stable cells were selected by FACS Aria I (BD).

\section{Fluorescence Microscopy}

For fluorescence microscopy, HeLa-iNap1-, HeLa-iNap3-Mit-, HeLa-mCherry-iNap1-, HeLa-mCherry-iNap3-Mit-, HeLa-SoNar-, HeLa-iNapc-, and HeLa-roGFP1-expressing 
cells were plated on a 4-well glass bottomed dish $\left(35 \mathrm{~mm}^{2}\right)$. Images were acquired using a high-performance fluorescence microscopy system equipped with a Nikon Eclipse Ti-E automatic microscope, monochrome cooled digital camera head DS-Qi1 Mc-U2, and the highly stable Sutter Lambda XL light source. A Plan Apo $60 \times 1.4$ NA water-immersion objective was utilized. The medium was changed to HBSS buffer (10 mM HEPES, 136.7 $\mathrm{mM} \mathrm{NaCl}, 5.4 \mathrm{mM} \mathrm{KCl}, 0.35 \mathrm{mM} \mathrm{Na}_{2} \mathrm{HPO}_{4}, 0.44 \mathrm{mM} \mathrm{KH}_{2} \mathrm{PO}_{4}, 4.2 \mathrm{mM} \mathrm{NaHCO}_{3}, 1.26$ $\mathrm{mM} \mathrm{CaCl}_{2}, 0.81 \mathrm{mM} \mathrm{MgSO}_{4}, \mathrm{pH}$ 7.4) with or without $10 \mathrm{mM}$ glucose. Cells were incubated for $1 \mathrm{hr}$ at $37{ }^{\circ} \mathrm{C}$ in a humidified atmosphere using a $\mathrm{CO}_{2}$ incubator (Tokai Hit) before imaging. For dual-excitation ratio imaging, $407 \mathrm{BP} 17 \mathrm{~nm}$, or $482 \mathrm{BP} 35 \mathrm{~nm}$, band-pass excitation filters (Semrock) and a $535 \mathrm{BP} 40 \mathrm{~nm}$, emission filter altered by a Lambda 10-XL filter wheel (Shutter Instruments) were used. For $\mathrm{pH}$-resistant ratiometric imaging of mCherry-iNap sensors, $407 \mathrm{BP} 17 \mathrm{~nm}$ or $560 \mathrm{BP} 40 \mathrm{~nm}$, excitation filters and $535 \mathrm{BP} 40 \mathrm{~nm}$ or $630 \mathrm{BP} 60 \mathrm{~nm}$, emission filters were used. Images were captured using $1280 \times 1024$ format, 12 bit depth, $2 \times$ gain. Raw data were exported to ImageJ software as 12 bit TIFF file for analysis. The pixel-by-pixel ratio of the $407 \mathrm{~nm}$ excitation image by the $482 \mathrm{~nm}$ excitation image of the same cell was used to pseudocolor the images in HSB color space. Simply, the RGB value $(255,0,255)$ represents the lowest ratio, and the red value $(255,0,0)$ represents the highest ratio, while the color brightness is proportional to the fluorescent signals in both channels. Images were taken on random, but cells with extraordinarily strong or low expression levels were excluded for examination.

For intracellular calcium imaging, cells were loaded with $5 \mu$ M Fluo-4 AM (Invitrogen) for $30 \mathrm{~min}$, rinsed twice with HBSS buffer, and then incubated in HBSS buffer. Images were captured using a Plan Apo $20 \times 0.75$ NA objective, 482 BP $35 \mathrm{~nm}$, excitation filter, $535 \mathrm{BP}$ $40 \mathrm{~nm}$, emission filter, $640 \times 480$ format, and 12 bit depth. Raw data were exported to ImageJ software as 12 bit TIF files for analysis. Images were taken on random, but cells with extraordinarily strong or low expression levels were excluded for examination.

\section{In Vitro mRNA Transcription and General Fish Imaging Procedures}

The gene of iNap1, iNapc and HyPerRed ${ }^{33}$ (Red $\mathrm{H}_{2} \mathrm{O}_{2}$ sensor) were subcloned into the pTol2 plasmid with SP6 promoter and a SV40 polyA terminator. After amplified by PCR, the mRNA was transcribed in vitro using the mMESSAGE mMACHINE kit (Qiagen) according to the manufacturer's manual. The DNA template was digested by addition of 2 units of DNase, followed by mRNA precipitation using lithium chloride incubation for 2 hours at $-20{ }^{\circ} \mathrm{C}$.

For the co-imaging of $\mathrm{H}_{2} \mathrm{O}_{2}$ and NADPH dynamics in zebrafish, we mixed equal part iNap1 and HyPerRed mRNA (the final concentration of $50 \mathrm{ng} / \mathrm{ml}$ for each mRNA) and microinjected to one-cell-stage zebrafish embryos with $1 \mathrm{nl}$. The mRNA of iNapc was diluted at the final concentration of $50 \mathrm{ng} / \mathrm{ml}$ and performed as a $\mathrm{pH}$ control. The handling procedures were approved by Institute of Neuroscience, Chinese Academy of Sciences.

The zebrafish embryos were maintained in egg water containing $0.2 \mathrm{mM} \mathrm{N}$-phenylthiourea (PTU; Sigma) to prevent pigment formation at $28{ }^{\circ} \mathrm{C}$ for 2 days under standard laboratory conditions. Zebrafish larvae were anaesthetized in E3 medium $(5 \mathrm{mM} \mathrm{NaCl}, 0.17 \mathrm{mM} \mathrm{KCl}$, $0.33 \mathrm{mM} \mathrm{CaCl}_{2}, 0.33 \mathrm{mM} \mathrm{MgSO}_{4}, 0.6 \mathrm{mM}$ Tricaine) and subjected to tail-fin tip amputation 
under stereoscopic microscope. Then the injured larvae were mounted using $1 \%$ low-melting agarose in a glass-bottom dish. To inhibit the activity of NADPH oxidase, we incubated larvae in E3 medium containing $0.1 \mathrm{mM}$ DPI (Sigma) $30 \mathrm{~min}$ before tail-fin tip amputation as described ${ }^{34}$. Fluorescence images of iNap1 or iNapc were captured using a Plan Apo 20 $\times 0.75$ NA objective, 425 BP $30 \mathrm{~nm}$ or 482 BP $35 \mathrm{~nm}$, excitation filters, 535 BP $40 \mathrm{~nm}$, emission filter, The HyPerRed signal was obtained using $560 \mathrm{BP} 40 \mathrm{~nm}$, excitation filter, 630 BP $60 \mathrm{~nm}$, emission filter. Image was processed as described above.

\section{Fluorescence-activated Cell Sorter Analysis (FACS)}

RAW264.7 cells were transiently transfected iNap1 sensors using FuGENE HD transfection reagent (Promega). To activate macrophages, LPS $(0.5 \mu \mathrm{g} / \mathrm{ml})$ plus IFN- $\gamma(250 \mathrm{U} / \mathrm{ml})$ were added 24 hours after transfection for 15 hours. Cells were scraped and monitored using FACS. FACS was performed with CytoFLEX-S flowcytometer (Beckman Coulter). Cells were excited using laser lines at $405 \mathrm{~nm}$ and $488 \mathrm{~nm}$. Emission filters were 525/40 for both excitation wavelengths.

\section{Live-Cell Fluorescence Measurement Using Microplate Reader}

Cells were counted and seeded into black 96-well flat bottomed plates with 30,000 cells per well. After 10-12 hr, cells were washed, and incubated in HBSS buffer, and treated with different compounds at $37^{\circ} \mathrm{C}$ during the measurement. Dual-excitation ratios were obtained by a Synergy 2 Multi-Mode Microplate Reader (BioTek) with excitation filters 420 BP 10 $\mathrm{nm}$, and $485 \mathrm{BP} 20 \mathrm{~nm}$, and emission filter $528 \mathrm{BP} 20 \mathrm{~nm}$ (for both excitation wavelengths). Dual-emission measurements were obtained with the excitation filter $420 \mathrm{BP} 10 \mathrm{~nm}$, (for both emission wavelengths), and the emission filters $485 \mathrm{BP} 20 \mathrm{~nm}$, and $528 \mathrm{BP} 20 \mathrm{~nm}$. Fluorescence values were background corrected by subtracting the intensity of the cell samples not expressing iNap or other sensors. Unless otherwise indicated, $10 \mathrm{mM}$ glucose was maintained in the buffer.

\section{Calibration of Intracellular NADPH Levels}

Intracellular NADPH levels can be measured after calibration of iNap fluorescence in live cells with that of recombinant iNap protein as described previously ${ }^{9}$. For the determination of the \% sensor occupation, we compared to the emission ratios of cells expressing sensors before and after treatment with digitonin in the presence or absence of high concentration of NADPH. For the digitonin permeabilization assay, iNap1-expressing cells were incubated in HEPES buffer ( $\mathrm{pH} 7.3$ for cytosol $^{45}$ ) containing $100 \mu \mathrm{g} / \mathrm{ml}$ digitonin. iNap3-Mit-expressing cells were incubated with HEPES buffer ( $\mathrm{pH} 8.0$ for mitochondrial matrix ${ }^{45}$ ) containing 1 $\mathrm{mg} / \mathrm{ml}$ digitonin; the fluorescence ratio corrected by iNapc of cells in the presence or absence of $0.2 \mathrm{mM}$ or $0.6 \mathrm{mM}$ NADPH was then measured in parallel to correct the $\mathrm{pH}$ effects.

\section{G6PD, PGD and NADK Activity Assay}

The enzymatic activity of glucose-6-phosphate dehydrogenase (G6PD) and 6phosphogluconate dehydrogenase (PGD) was measured with MTT-based cycling methods as described previously ${ }^{46}$. Briefly, $\mathrm{NADP}^{+}$is converted to NADPH by G6PD and PGD and 
then recycled by reduction of MTT to formazan, which can be quantified by measuring the absorbance at $600 \mathrm{~nm}$. Because the product of G6PD is hydrolyzed to the substrate for PGD, an accurate activity of G6PD is obtained from the difference of the PGD activity and the combined activity. For the total activity assay, cell lysate were added to the reaction buffer (50 mM Tris- $\mathrm{HCl}, 1 \mathrm{mM} \mathrm{MgCl} 2,0.2 \mathrm{mM}$ glucose-6-phosphate, $0.2 \mathrm{mM}$ 6phosphogluconate, $0.1 \mathrm{mM} \mathrm{NADP}^{+}, 0.5 \mathrm{mM}$ MTT, $1.5 \mathrm{mM}$ phenazine methosulfate, $\mathrm{pH}$ 8.0), and the catalysis of PGD was measured in the same reaction buffer without G6P. The activity was normalized to protein concentration.

The NADK activity assay was performed using a two-step procedure described previously ${ }^{47}$. $\mathrm{NAD}^{+}$was firstly phosphorylated to $\mathrm{NADP}^{+}$by NADK and then measured as described above. Specifically, cell lysates were added to a $10 \mu \mathrm{l}$ reaction solution $(50 \mathrm{mM}$ Tris- $\mathrm{HCl}, 10$ $\mathrm{mM} \mathrm{MgCl} 2,5 \mathrm{mM} \mathrm{NAD}{ }^{+}, 10 \mathrm{mM}$ ATP, $\mathrm{pH} 7.8$ ) and incubated at $30{ }^{\circ} \mathrm{C}$ for $5 \mathrm{~min}$. The mixture was then transferred to a $90 \mu$ cycling buffer ( $50 \mathrm{mM}$ Tris- $\mathrm{HCl}, 5 \mathrm{mM} \mathrm{MgCl}, 5$ mM G6P, $0.5 \mathrm{mM}$ MTT, $1.5 \mathrm{mM}$ PMS and $2 \mathrm{unit} / \mathrm{ml}$ yeast G6PD, $\mathrm{pH}$ 8.0). The absorbance at $600 \mathrm{~nm}$ was monitored by microplate reader. All samples were run in triplicate.

\section{NADPH Assay}

The intracellular levels of NADPH were determined by the enzymatic cycling method ${ }^{48}$. In brief, $2 \times 10^{6}$ cells were lysed with $0.4 \mathrm{ml}$ of extraction buffer ( $20 \mathrm{mM} \mathrm{NAM}, 20 \mathrm{mM}$ $\mathrm{NaHCO}_{3}, 100 \mathrm{mM} \mathrm{Na} 2 \mathrm{CO}_{3}$ ) by performing a freeze/thaw cycle (20 min on dry ice followed by $10 \mathrm{~min}$ at room temperature). The lysate was centrifuged at $12,000 \mathrm{rpm}$ for $10 \mathrm{~min}$, and the supernatant was incubated in a heating block for $30 \mathrm{~min}$ at $65^{\circ} \mathrm{C}$ to decompose $\mathrm{NADP}^{+}$. The heated sample $(10 \mu \mathrm{l})$ was added to $90 \mu \mathrm{l}$ NADP-cycling buffer ( $100 \mathrm{mM}$ Tris-HCl, 0.5 $\mathrm{mM}$ thiazolylblue, $1.5 \mathrm{mM}$ phenazine ethosulfate, $5 \mathrm{mM}$ EDTA, $1 \mathrm{mM} \mathrm{G6P}$ and $2 \mathrm{unit} / \mathrm{ml}$ $\mathrm{G} 6 \mathrm{PD}, \mathrm{pH}$ 8.0). The absorbance changes at $600 \mathrm{~nm}$ were measured every $30 \mathrm{sec}$ for $10 \mathrm{~min}$ at $30{ }^{\circ} \mathrm{C}$ using a Synergy 2 multi-mode microplate reader. Mitochondria were extracted using Cell Mitochondria Isolation kit (Beyotime Biotechnology, China) according to the manual. Mitochondrial NADPH was quantified as described above and normalized to the protein concentration.

\section{Western Blot}

For the Western blot analyses, cells were lysed in $1 \times$ SDS sample buffer supplemented with a protease/phosphatase inhibitor cocktail (Cell Signaling Technology). Equal amounts of total protein $(30-50 \mu \mathrm{g})$ were separated on sodium dodecyl sulfate-polyacrylamide gel electrophoresis (SDS-PAGE), and electrotransferred onto PVDF membranes. Membranes were incubated with primary antibodies to: NADK (sc-100347), G6PD (sc-373886), PGD (sc-398977), actin (sc-1616) and GAPDH (sc-365062) (Santa Cruz Biotechnologies), phospho-AMPKa (Thr172) (\#2531s), AMPKa(\#2532s), phospho-ACC (Ser79) (\#3661s), and ACC(\#3676s) (Cell Signaling Technology), followed by secondary antibodies conjugated to horseradish peroxidase, addition of chemiluminescence detection mixture (Roche) and imaging. 


\section{Cell Viability Assay}

HeLa cells were plated at a density of $1 \times 10^{5}$ cells in 12 -well plates. After $\sim 20 \mathrm{~h}$, cells were pretreated with $2 \mu \mathrm{M}$ ionomycin, $200 \mu \mathrm{M}$ DHEA, or $10 \mu \mathrm{M}$ dorsomorphin for $1 \mathrm{hr}$, exposed to a 1-hr pulse of $200 \mu \mathrm{M}$ or $300 \mu \mathrm{M}$ diamide, then grown for $24 \mathrm{hr}$. Cell survival was determined using a Countstar automated cell counter (Inno-Alliance Biotech Inc., Wilmington, USA).

\section{Statistical Analysis}

Data are presented either as a representative example of a single experiment repeated at least in triplicate or as three or more experiments. Data obtained are represented as mean values \pm $\mathrm{SD}$. or mean values \pm SEM. All $\mathrm{p}$ values were obtained using unpaired two-tailed Student's $t$ test. Values of $\mathrm{p}<0.05$ were considered statistically significant $(* \mathrm{p}<0.05, * * \mathrm{p}<0.01$, and $* * * \mathrm{p}<0.001)$

\section{Data Availability}

The data that supports the conclusions of this study are available from the corresponding author upon request. All constructs, including iNap1-4 and iNapc, are available on request.

\section{Supplementary Material}

Refer to Web version on PubMed Central for supplementary material.

\section{Acknowledgments}

We thank S. James Remington for the roGFP1 vector; Gary Yellen for the PercevalHR vector; Vsevolod V. Belousov for the HyPerRed vector. Chun Fan, Junnan Ye, Yun Tang, Tianduanyi Wang, Zengrui Wu, Hanyang Hu, Zengmin Du, Ting Li, Yuelan Xia, Li zhou and Yuxiao Wang for technical assistance; and Stephanie Tribuna for secretarial assistance. This research was supported by the NSFC (31225008, 91313301, and 31470833 to Y.Y., 31370755 to H.L., 91649123 and 31671484 to Y. Zhao), 973 Program (2013CB531200 to Y.Y.), the Shanghai Science and Technology Commission (14XD1401400 and 16430723100 to Y.Y., and 15YF1402600 to Y. Zhao), the Specialized Research Fund for the Doctoral Program of Higher Education (20100074110010 to Y.Y.), the Lift Engineering for Young Talent of China Association for Science and Technology (to Y. Zhao), Shanghai Young Topnotch Talent (to Y. Zhao), the State Key Laboratory of Bioreactor Engineering (to Y.Y.), the 111 Project (B07023 to Y.Y.), the Fundamental Research Funds for the Central Universities (to Y.Y. and Y. Zhao) and the National Institutes of Health (grants HL061795, HL007690, and GM107618 to J.L.).

\section{References}

1. Gorrini C, Harris IS, Mak TW. Modulation of oxidative stress as an anticancer strategy. Nat Rev Drug Discov. 2013; 12:931-947. [PubMed: 24287781]

2. Schulze A, Harris AL. How cancer metabolism is tuned for proliferation and vulnerable to disruption. Nature. 2012; 491:364-373. [PubMed: 23151579]

3. Vallance P, Leiper J. Blocking NO synthesis: how, where and why? Nat Rev Drug Discov. 2002; 1:939-950. [PubMed: 12461516]

4. Trachootham D, Alexandre J, Huang P. Targeting cancer cells by ROS-mediated mechanisms: a radical therapeutic approach? Nat Rev Drug Discov. 2009; 8:579-591. [PubMed: 19478820]

5. Pollak N, Niere M, Ziegler M. NAD kinase levels control the NADPH concentration in human cells. J Biol Chem. 2007; 282:33562-33571. [PubMed: 17855339]

6. Nobrega-Pereira S, et al. G6PD protects from oxidative damage and improves healthspan in mice. Nat Commun. 2016; 7:10894. [PubMed: 26976705] 
7. MacDonald MJ. Feasibility of a mitochondrial pyruvate malate shuttle in pancreatic islets. Further implication of cytosolic NADPH in insulin secretion. J Biol Chem. 1995; 270:20051-20058. [PubMed: 7650022]

8. Blacker TS, et al. Separating NADH and NADPH fluorescence in live cells and tissues using FLIM. Nat Commun. 2014; 5:3936. [PubMed: 24874098]

9. Zhao Y, et al. Genetically encoded fluorescent sensors for intracellular NADH detection. Cell Metab. 2011; 14:555-566. [PubMed: 21982715]

10. Zhao Y, et al. SoNar, a highly responsive NAD+/NADH sensor, allows high-throughput metabolic screening of anti-tumor agents. Cell Metab. 2015; 21:777-789. [PubMed: 25955212]

11. Bilan DS, et al. Genetically encoded fluorescent indicator for imaging NAD/NADH ratio changes in different cellular compartments. Biochim Biophys Acta. 2013; 1840:951-957. [PubMed: 24286672]

12. Hung YP, Albeck JG, Tantama M, Yellen G. Imaging cytosolic NADH-NAD(+) redox state with a genetically encoded fluorescent biosensor. Cell Metab. 2011; 14:545-554. [PubMed: 21982714]

13. Cambronne XA, et al. Biosensor reveals multiple sources for mitochondrial NAD(+). Science. 2016; 352:1474-1477. [PubMed: 27313049]

14. Cameron WD, et al. Apollo-NADP(+): a spectrally tunable family of genetically encoded sensors for NADP(+). Nat Methods. 2016; 13:352-358. [PubMed: 26878383]

15. Zhao Y, et al. In vivo monitoring of cellular energy metabolism using SoNar, a highly responsive sensor for NAD(+)/NADH redox state. Nat Protoc. 2016; 11:1345-1359. [PubMed: 27362337]

16. Zhao Y, Yang Y. Real-time and high-throughput analysis of mitochondrial metabolic states in living cells using genetically encoded NAD+/NADH sensors. Free Radic Biol Med. 2016; 100:4352. [PubMed: 27261194]

17. Powers R, et al. Comparison of protein active site structures for functional annotation of proteins and drug design. Proteins. 2006; 65:124-135. [PubMed: 16862592]

18. Arai R, et al. Structure of archaeal glyoxylate reductase from Pyrococcus horikoshii OT3 complexed with nicotinamide adenine dinucleotide phosphate. Acta Crystallographica. 2007; 63:357-365. [PubMed: 17327673]

19. Nagai T, Sawano A, Park ES, Miyawaki A. Circularly permuted green fluorescent proteins engineered to sense Ca2+ Proc Natl Acad Sci U S A. 2001; 98:3197-3202. [PubMed: 11248055]

20. Hedeskov CJ, Capito K, Thams P. Cytosolic ratios of free [NADPH]/[NADP+] and [NADH]/[NAD $+]$ in mouse pancreatic islets, and nutrient-induced insulin secretion. Biochem J. 1987; 241:161167. [PubMed: 3551925]

21. Veech RL, Eggleston LV, Krebs HA. The redox state of free nicotinamide-adenine dinucleotide phosphate in the cytoplasm of rat liver. Biochem J. 1969; 115:609-619. [PubMed: 4391039]

22. Ohashi K, Kawai S, Murata K. Identification and characterization of a human mitochondrial NAD kinase. Nat Commun. 2012; 3:1248. [PubMed: 23212377]

23. Tantama M, Martinez-Francois JR, Mongeon R, Yellen G. Imaging energy status in live cells with a fluorescent biosensor of the intracellular ATP-to-ADP ratio. Nat Commun. 2013; 4:2550. [PubMed: 24096541]

24. Noda T, Amano F. Differences in nitric oxide synthase activity in a macrophage-like cell line, RAW264.7 cells, treated with lipopolysaccharide (LPS) in the presence or absence of interferongamma (IFN-gamma): possible heterogeneity of iNOS activity. J Biochem. 1997; 121:38-46. [PubMed: 9058189]

25. Takanaga H, Chaudhuri B, Frommer WB. GLUT1 and GLUT9 as major contributors to glucose influx in HepG2 cells identified by a high sensitivity intramolecular FRET glucose sensor. Biochim Biophys Acta. 2008; 1778:1091-1099. [PubMed: 18177733]

26. Dooley CT, et al. Imaging dynamic redox changes in mammalian cells with green fluorescent protein indicators. J Biol Chem. 2004; 279:22284-22293. [PubMed: 14985369]

27. Pandolfi PP, et al. Targeted disruption of the housekeeping gene encoding glucose 6-phosphate dehydrogenase (G6PD): G6PD is dispensable for pentose synthesis but essential for defense against oxidative stress. EMBO J. 1995; 14:5209-5215. [PubMed: 7489710] 
28. Au SW, Gover S, Lam VM, Adams MJ. Human glucose-6-phosphate dehydrogenase: the crystal structure reveals a structural NADP $(+)$ molecule and provides insights into enzyme deficiency. Structure. 2000; 8:293-303. [PubMed: 10745013]

29. Woods A, et al. Ca2+/calmodulin-dependent protein kinase kinase-beta acts upstream of AMPactivated protein kinase in mammalian cells. Cell Metab. 2005; 2:21-33. [PubMed: 16054096]

30. Jeon SM, Chandel NS, Hay N. AMPK regulates NADPH homeostasis to promote tumour cell survival during energy stress. Nature. 2012; 485:661-665. [PubMed: 22660331]

31. Tsou P, Zheng B, Hsu CH, Sasaki AT, Cantley LC. A fluorescent reporter of AMPK activity and cellular energy stress. Cell Metab. 2011; 13:476-486. [PubMed: 21459332]

32. Oakhill JS, et al. AMPK is a direct adenylate charge-regulated protein kinase. Science. 2011; 332:1433-1435. [PubMed: 21680840]

33. Ermakova YG, et al. Red fluorescent genetically encoded indicator for intracellular hydrogen peroxide. Nat Commun. 2014; 5:5222. [PubMed: 25330925]

34. Niethammer P, Grabher C, Look AT, Mitchison TJ. A tissue-scale gradient of hydrogen peroxide mediates rapid wound detection in zebrafish. Nature. 2009; 459:996-999. [PubMed: 19494811]

35. Belousov VV, et al. Genetically encoded fluorescent indicator for intracellular hydrogen peroxide. Nat Methods. 2006; 3:281-286. [PubMed: 16554833]

36. Berg J, Hung YP, Yellen G. A genetically encoded fluorescent reporter of ATP:ADP ratio. Nat Methods. 2009; 6:161-166. [PubMed: 19122669]

37. Pomorski A, Kochanczyk T, Miloch A, Krezel A. Method for Accurate Determination of Dissociation Constants of Optical Ratiometric Systems: Chemical Probes, Genetically Encoded Sensors, and Interacting Molecules. Analytical Chemistry. 2013; 85:11479-11486. [PubMed: 24180305]

38. Pavlova NN, Thompson CB. The Emerging Hallmarks of Cancer Metabolism. Cell Metab. 2016; 23:27-47. [PubMed: 26771115]

39. Cool B, et al. Identification and characterization of a small molecule AMPK activator that treats key components of type 2 diabetes and the metabolic syndrome. Cell Metab. 2006; 3:403-416. [PubMed: 16753576]

40. Yavari A, et al. Chronic Activation of gamma2 AMPK Induces Obesity and Reduces beta Cell Function. Cell Metab. 2016; 23:821-836. [PubMed: 27133129]

41. Burkewitz K, Zhang Y, Mair WB. AMPK at the nexus of energetics and aging. Cell Metab. 2014; 20:10-25. [PubMed: 24726383]

42. Shackelford DB, Shaw RJ. The LKB1-AMPK pathway: metabolism and growth control in tumour suppression. Nat Rev Cancer. 2009; 9:563-575. [PubMed: 19629071]

43. Thliveris AT, Little JW, Mount DW. Repression of the E coli recA gene requires at least two LexA protein monomers. Biochimie. 1991; 73:449-456. [PubMed: 1911945]

44. Zhao Y, et al. An expanded palette of genetically encoded $\mathrm{Ca}(2)(+)$ indicators. Science. 2011; 333:1888-1891. [PubMed: 21903779]

45. Llopis J, McCaffery JM, Miyawaki A, Farquhar MG, Tsien RY. Measurement of cytosolic, mitochondrial, and Golgi $\mathrm{pH}$ in single living cells with green fluorescent proteins. Proc Natl Acad Sci U S A. 1998; 95:6803-6808. [PubMed: 9618493]

46. Tian WN, et al. Importance of glucose-6-phosphate dehydrogenase activity for cell growth. J Biol Chem. 1998; 273:10609-10617. [PubMed: 9553122]

47. Outten CE, Culotta VC. A novel NADH kinase is the mitochondrial source of NADPH in Saccharomyces cerevisiae. EMBO J. 2003; 22:2015-2024. [PubMed: 12727869]

48. Wagner TC, Scott MD. Single extraction method for the spectrophotometric quantification of oxidized and reduced pyridine nucleotides in erythrocytes. Anal Biochem. 1994; 222:417-426. [PubMed: 7864367] 


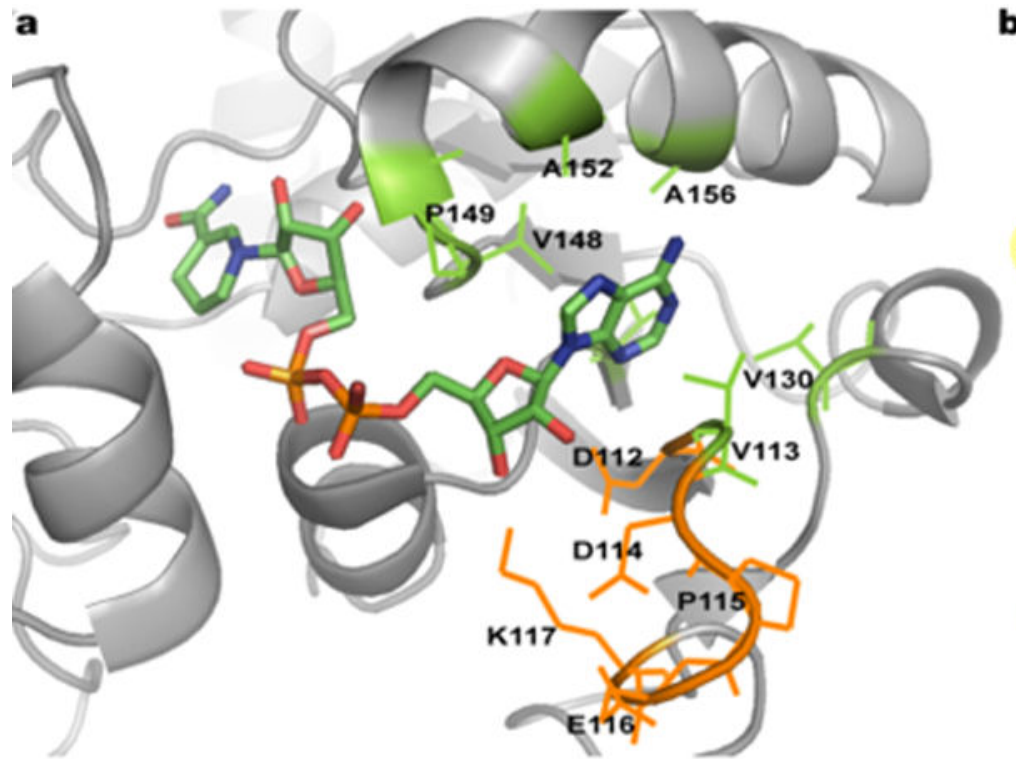

b
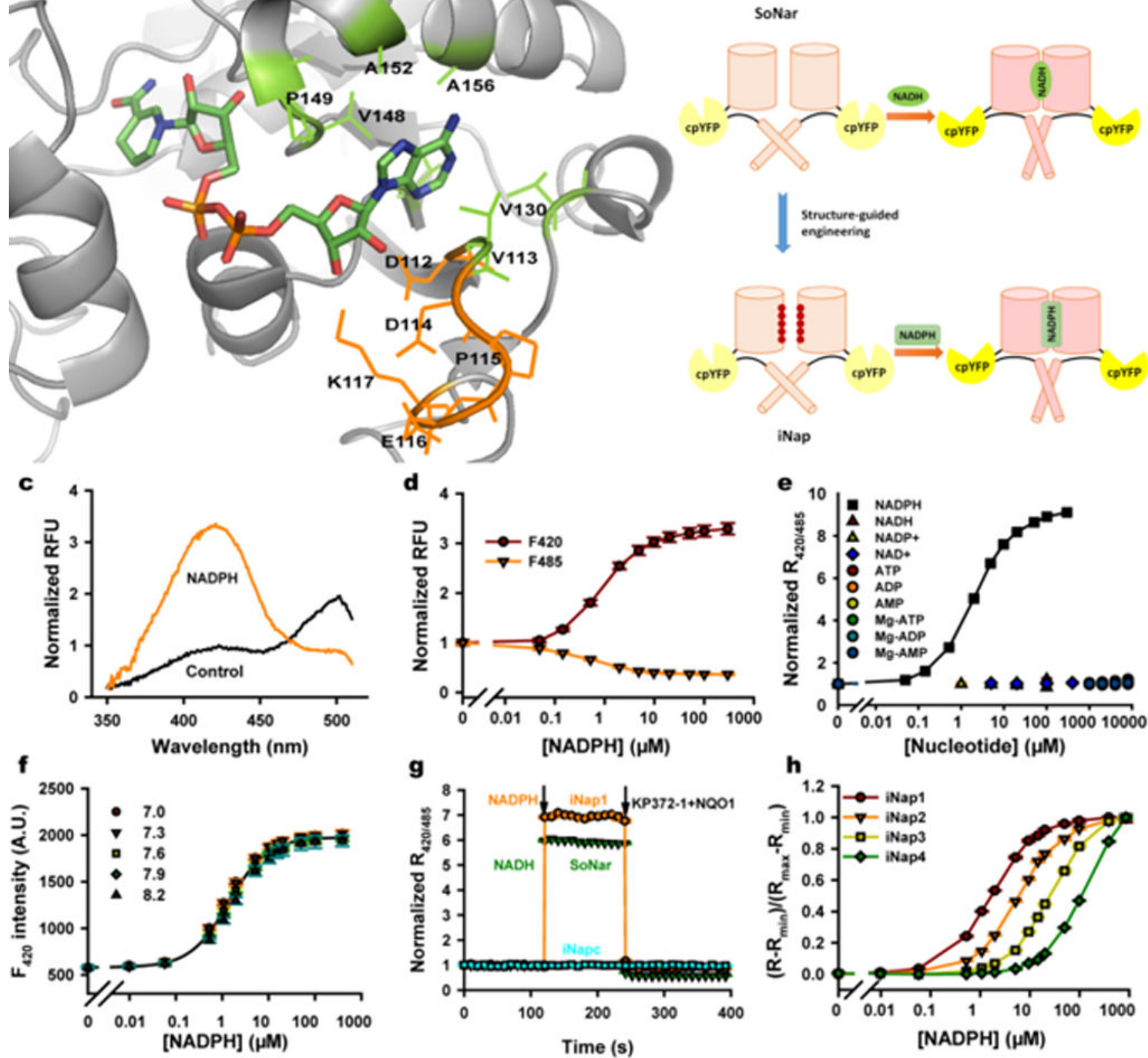

Figure 1. Structure-guided engineering and characterization of the iNap sensors

(a) The NADH binding site of the T-Rex, target for molecular engineering (PDB ID:1xcb). It contains a charged selective loop (orange) around the 2'-hydroxyl functionality of the ligand and a hydrophobic adenine-binding pocket (green). (b) The working mechanism of iNap and SoNar sensors. (c) Excitation spectra of purified iNap1 in the control condition (black), and after addition of $100 \mu \mathrm{M}$ NADPH (orange), normalized to the $420 \mathrm{~nm}$ peak intensity in the control condition. Emission was measured at $530 \mathrm{~nm}$. (d) Fluorescence intensities of iNap1 with excitation at $420 \mathrm{~nm}$ or $485 \mathrm{~nm}$ in the presence of different concentrations of NADPH, and emission at $528 \mathrm{~nm}$. Data are normalized to the initial value. (e) The ratio of fluorescence intensities with excitation at $420 \mathrm{~nm}$ divided by $485 \mathrm{~nm}\left(\mathrm{R}_{420 / 485}\right)$ in the 
presence of different concentrations of NADPH and its analogs. (f) Fluorescence intensity of iNap1 when excited at $420 \mathrm{~nm}$ plotted against the NADPH concentration at the indicated $\mathrm{pH}$. (g) Kinetics of fluorescence response of purified iNap1 and SoNar protein to $10 \mu \mathrm{M}$ NADPH and $10 \mu \mathrm{M}$ NADH, respectively. NADPH or NADH was subsequently decreased (oxidized) by the addition of redox cycling agents containing $1.5 \mu \mathrm{M} \mathrm{KP} 372-1$ and $3 \mu \mathrm{M}$ NQO1 protein. (h) NADPH titration curves of iNap1-4, with binding affinities were determined as $2 \mu \mathrm{M}, 6 \mu \mathrm{M}, 25 \mu \mathrm{M}$ and $120 \mu \mathrm{M}$, respectively. In (d)-(h) Data were represented as mean \pm s.e.m. 

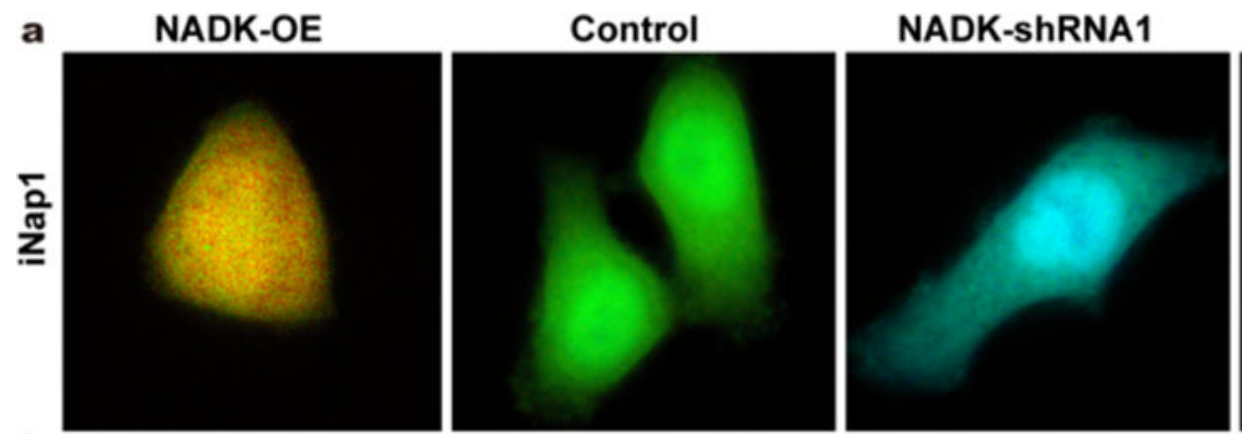

\section{NADK-ShRNA2}
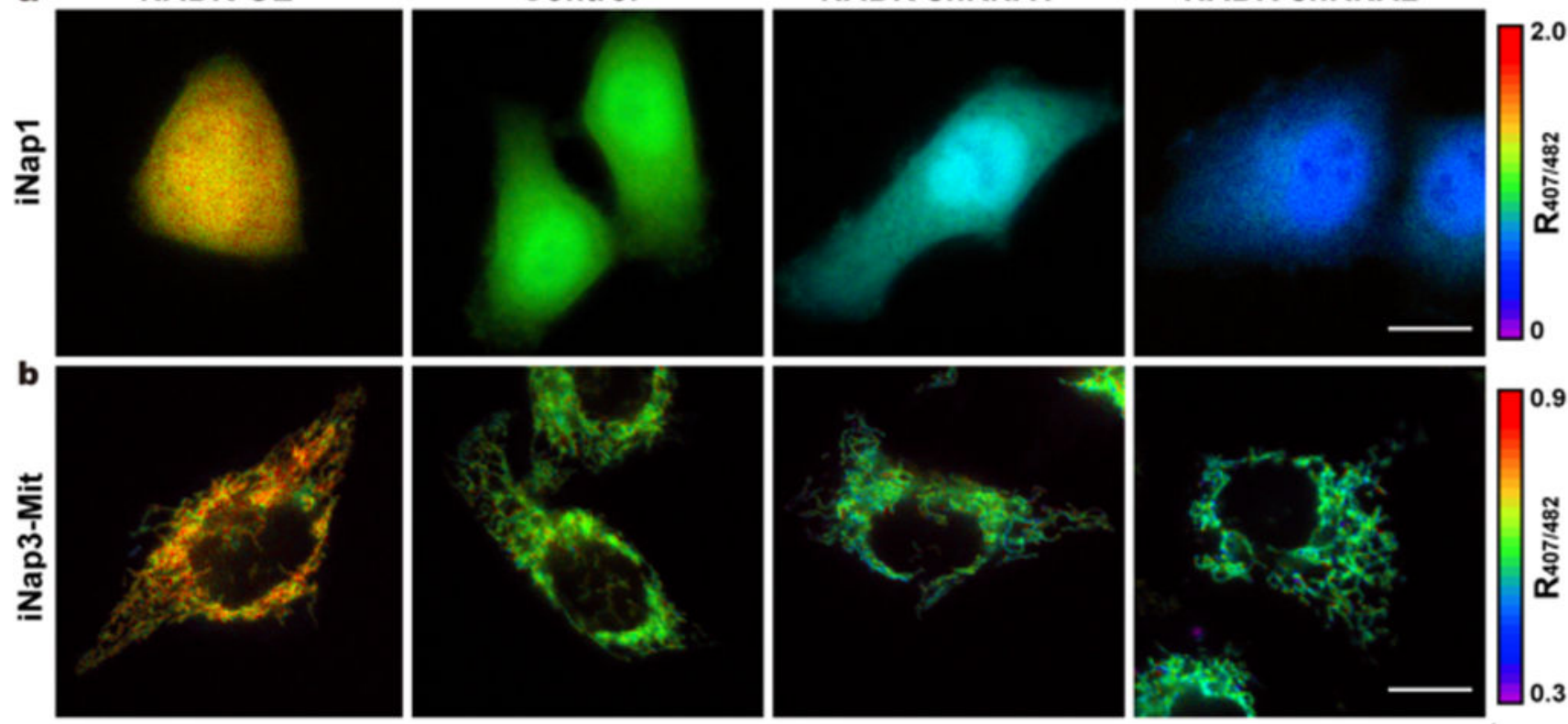

c
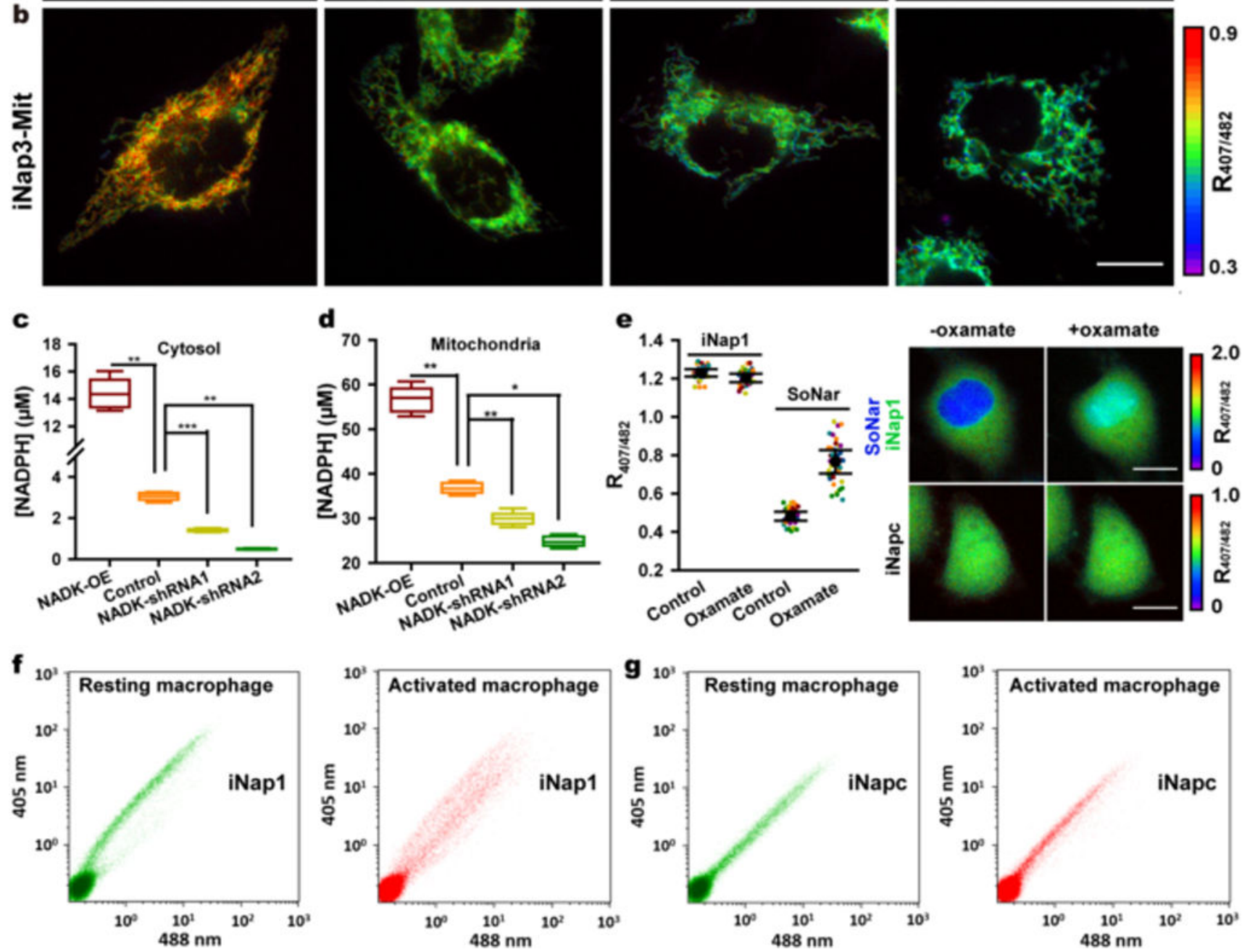

Figure 2. Subcellular distribution and regulation of NADPH in mammalian cells (a and b) Fluorescence images of iNap1 (a) or iNap3-Mit (b) in wide-type, NADKoverexpressing, and NADK-deficient HeLa cells. Images were pseudocolored with the ratio of fluorescence excited at $407 \mathrm{~nm}$ and $482 \mathrm{~nm}$. Scale bar, $10 \mu \mathrm{m}$. (c and d) Quantification of iNap1 (c) and iNap3-Mit (d) fluorescence in NADK overexpressing or knockdown cells. Data are normalized to digitonin-permeabilized cell samples in the absence of NADPH. Unpaired t-test. $* \mathrm{p}<0.05, * * \mathrm{p}<0.01$, and $* * * \mathrm{p}<0.001$. (e) Averaged ratio changes (left, 40 cells) and multiparametric imaging (right) of HeLa cells simultaneously expressing nucleus-localized SoNar and nucleus-excluded iNap1 in response to the LDH inhibitor 
oxamate. iNapc-expressing HeLa cells as a control. Scale bar $10 \mu \mathrm{M}$. Data are represented as mean \pm s.d. (f) FACS measurements of iNap1 fluorescence with excitation at $405 \mathrm{~nm}$ or 488 $\mathrm{nm}$ in resting or activated RAW264.7 mouse macrophages. (g) iNapc fluorescence in resting or activated RAW264.7 mouse macrophages measured with flow cytometer. 

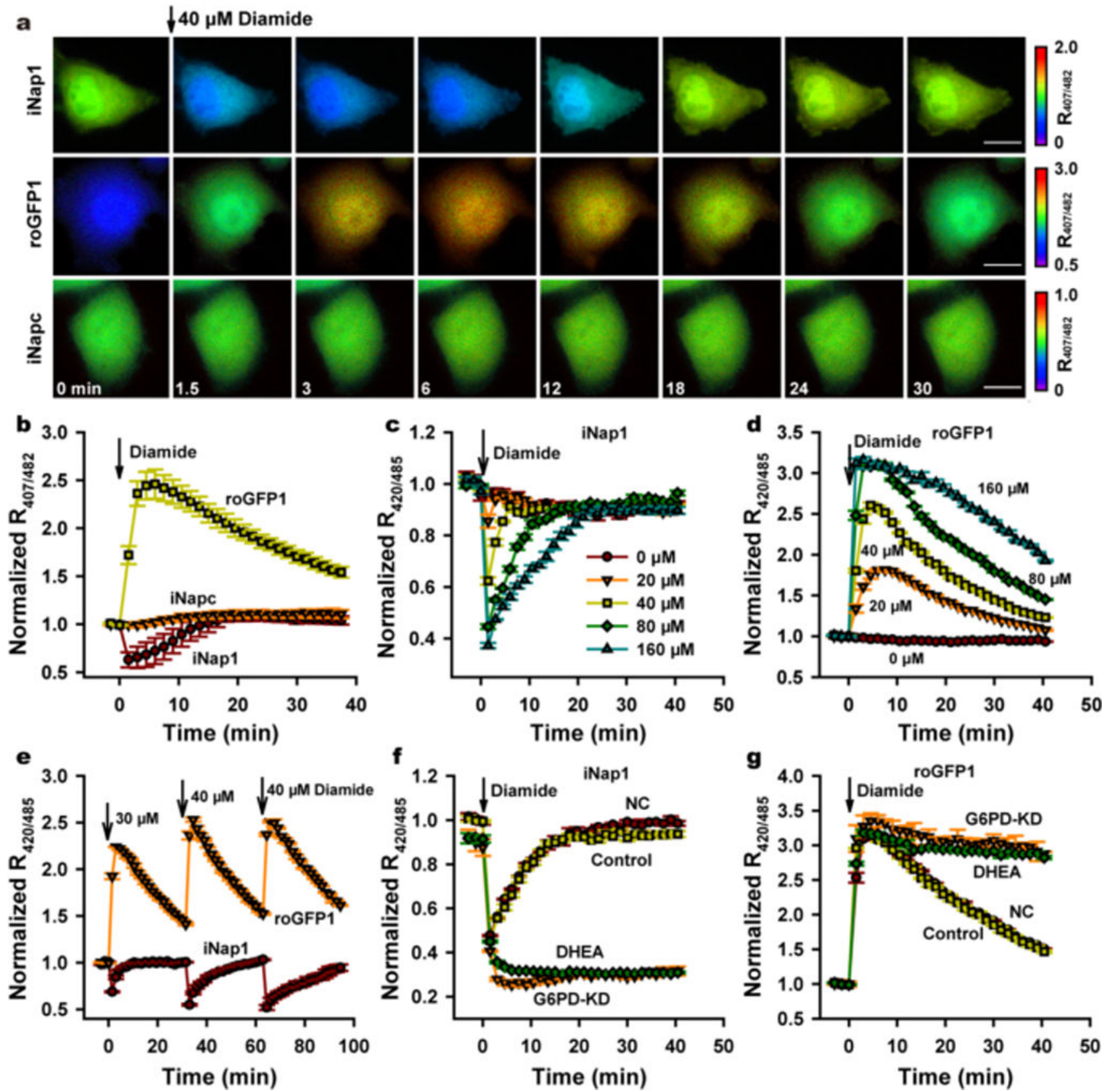

Figure 3. NADPH dynamics of glucose-deprived cells in the setting of oxidative stress

(a) Spatiotemporally resolved changes in the ratiometric fluorescence of iNap1, roGFP1, or iNapc in sequential frames (left to right) in response to $40 \mu \mathrm{M}$ diamide in HeLa cells. Images are pseudocolored with the ratio of fluorescence excited at $407 \mathrm{~nm}$ and $482 \mathrm{~nm}$. Scale bar, $10 \mu \mathrm{m}$. (b) Kinetics of iNap1, roGFP1, or iNapc fluorescence in HeLa cells in response to $40 \mu \mathrm{M}$ diamide. (c and d) Kinetics of iNap1 (c) or roGFP1 (d) fluorescence responses in HeLa cells upon addition of the indicated concentrations of diamide. (e) Kinetics of iNap1 and roGFP1 fluorescence in HeLa cells in response to three successive additions of diamide. (f and g) Kinetics of iNap1 (f) or roGFP1 (g) fluorescence in G6PD- 
knockdown or DHEA-treated HeLa cells in response to diamide. All experiments were performed in glucose-deprived HeLa cells and data are normalized to samples without diamide addition. For panel Fig. 3c, e, f, "R" represents Ratio of iNap1 corrected by iNapc. Data are represented as mean \pm s.e.m. 

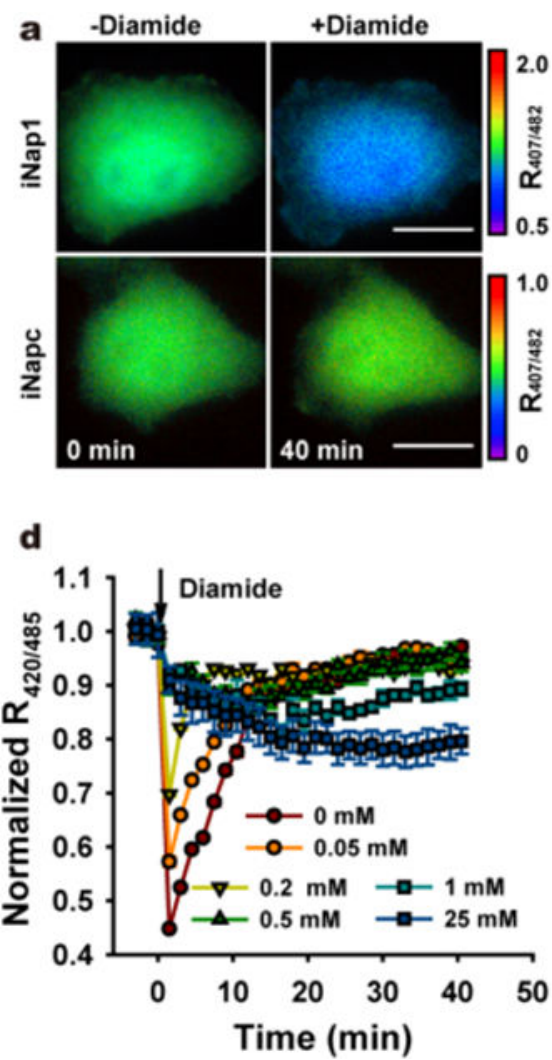

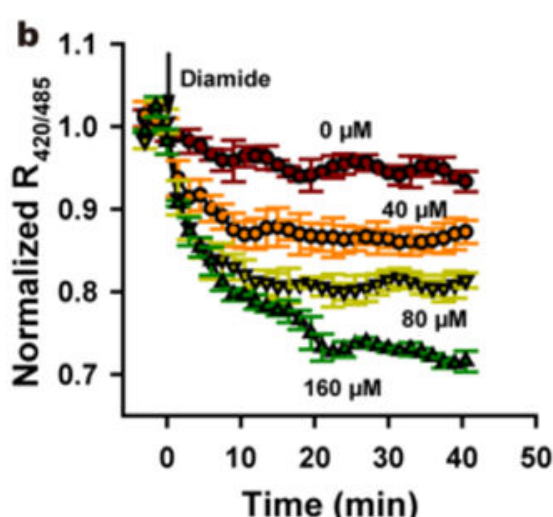

e

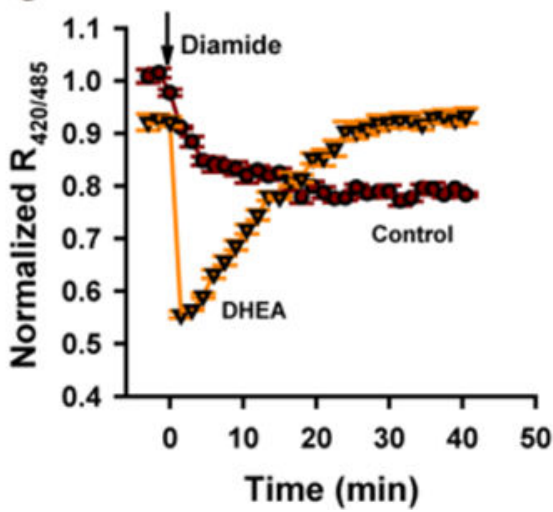

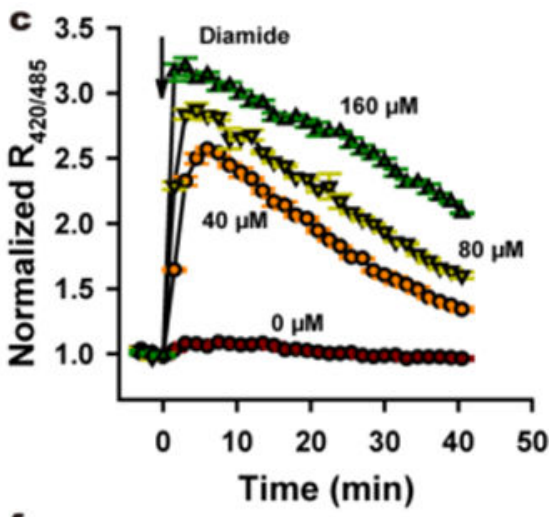

f

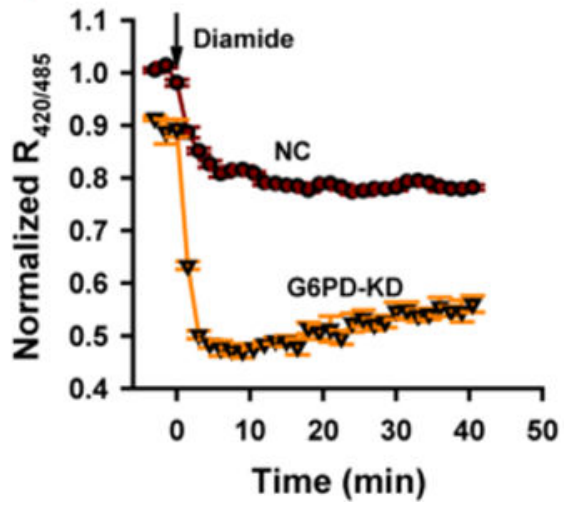

Figure 4. NADPH dynamics of glucose-fed cells in the setting of oxidative stress

(a) Fluorescence image of iNap1 or iNapc-expressing HeLa cells before and 40 min after treatment with $40 \mu \mathrm{M}$ diamide. Images are pseudocolored with the ratio of the $407 \mathrm{~nm}$ excitation image to the $482 \mathrm{~nm}$ excitation images of the same cell. Scale bar, $10 \mu \mathrm{m}$. (b and c) Kinetics of iNap1 (b) or roGFP1 (c) fluorescence responses in HeLa cells upon addition of the indicated concentrations of diamide. (d) Fluorescence response of iNap1 to $80 \mu \mathrm{M}$ diamide at different concentrations of glucose. (e and f) Kinetics of iNap1 fluorescence in DHEA-treated (e) or G6PD-knockdown (f) HeLa cells in response to $80 \mu \mathrm{M}$ diamide. All experiments were performed in glucose-fed HeLa cells unless otherwise indicated, and data are normalized to samples without diamide addition. For panel Fig. 4b, d-f, "R" represents ratio of iNap1 corrected by iNapc. Data are represented as mean \pm s.e.m. 


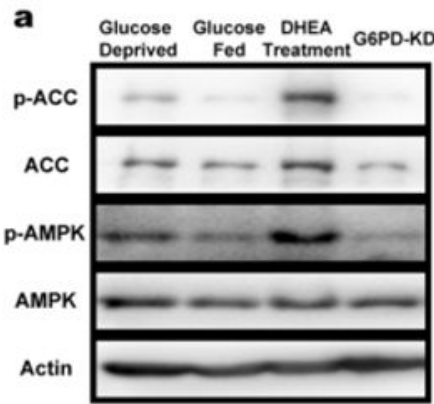

d

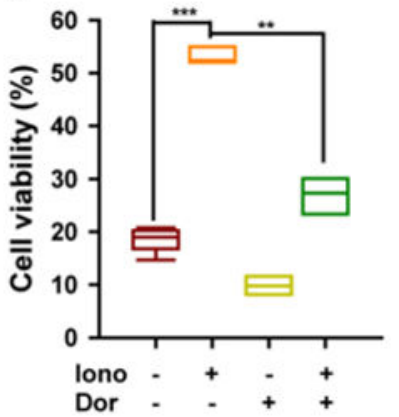

$\mathbf{g}$

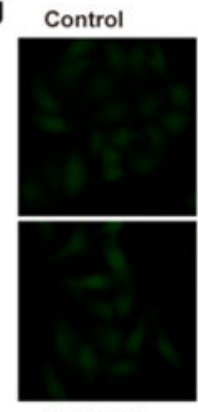

G6PD-KD

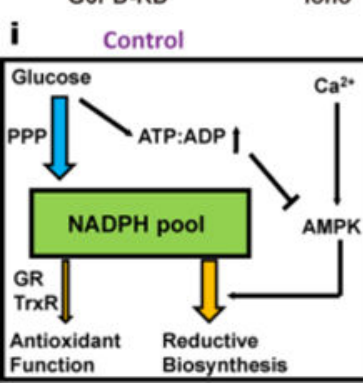

Iono

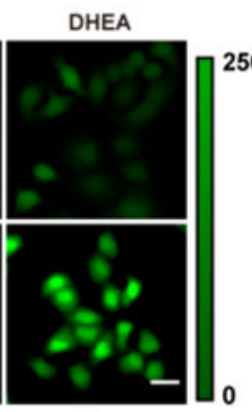

j Oxidative Stress
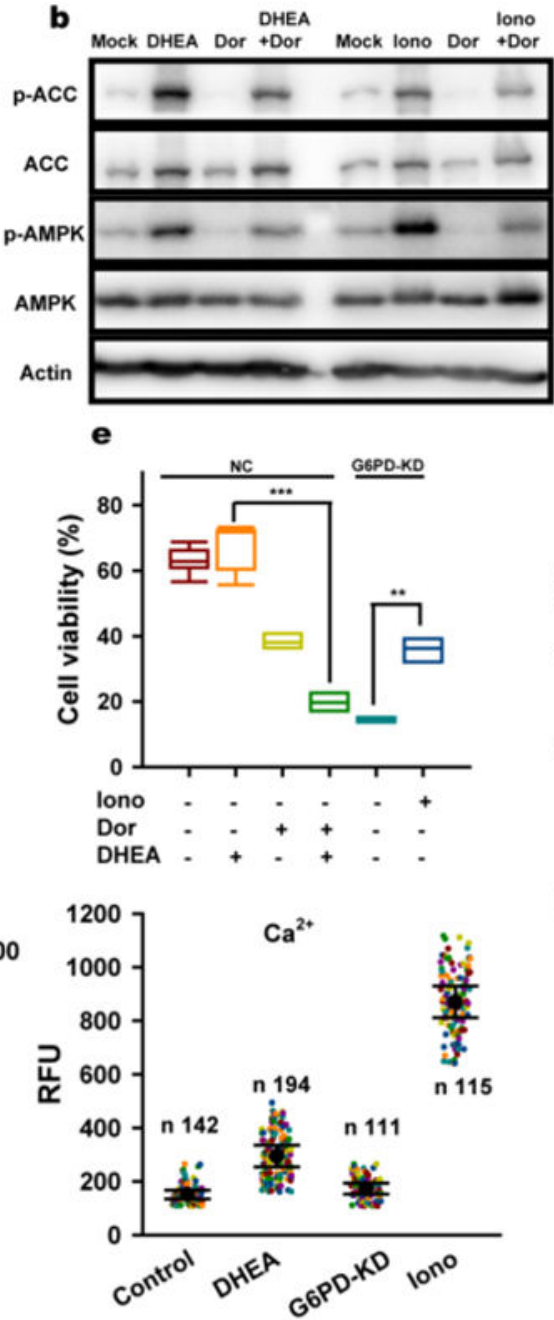
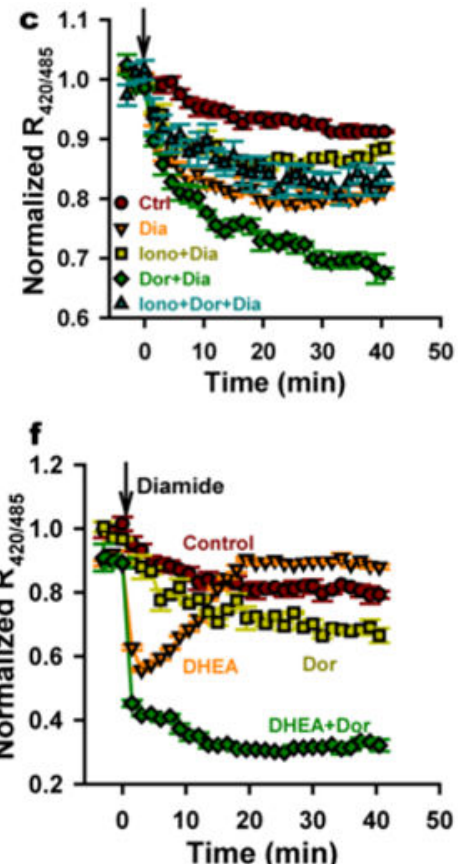

h

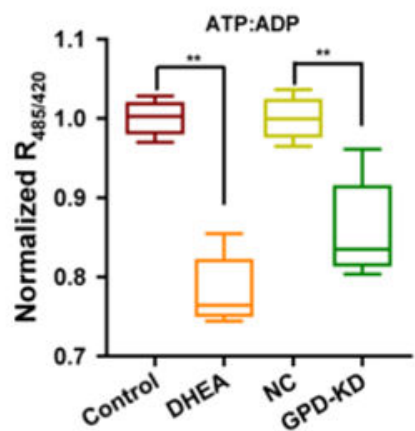

Figure 5. AMPK activation protects against oxidative stress-induced NADPH oxidation and cell death

(a) Phosphorylation of AMPK (T172) and AMPK substrate ACC (S79) in glucose-fed cells, glucose-deprived cells, DHEA-treated cells, and G6PD knockdown cells. (b) The effect of $200 \mu \mathrm{M}$ DHEA, $10 \mu \mathrm{M}$ dorsomorphin (AMPK inhibitor, Dor) and $0.5 \mu \mathrm{M}$ ionomycin (AMPK activator, Iono) on phosphorylation of ACC and AMPK in glucose-fed cells. (c) The effect of ionomycin on diamide-induced NADPH oxidation. Cells were treated with $0.5 \mu \mathrm{M}$ ionomycin for $15 \mathrm{~min}$, followed by $80 \mu \mathrm{M}$ diamide. (d) The effect of ionomycin on diamideinduced cell death. Cells were exposed to a 1-hr pulse of $300 \mu \mathrm{M}$ diamide either with or 
without $2 \mu \mathrm{M}$ ionomycin and then grown for $24 \mathrm{hr}$. (e) The effect of DHEA on diamideinduced cell death. Cells were exposed to a 1-hr pulse of $200 \mu \mathrm{M}$ diamide either with or without $200 \mu \mathrm{M}$ DHEA and then grown for $24 \mathrm{hr}$. For 5d, e, Unpaired t-test. $* * \mathrm{p}<0.01$, and $* * * \mathrm{p}<0.001$. (f) The effect of dorsomorphin on diamide-induced NADPH oxidation in DHEA-treated cells. Cells were treated with $200 \mu \mathrm{M}$ DHEA for $60 \mathrm{~min}$, followed by $80 \mu \mathrm{M}$ diamide. (g) Fluorescence images (left) and relative levels (right) of intracellular $\mathrm{Ca}^{2+}$ in glucose-fed cells, DHEA treated cells, G6PD knockdown cells, and ionomycin treated cells. Cells were loaded with the $\mathrm{Ca}^{2+}$ indicator Fluo-4 AM. Data were represented as mean \pm s.d. (h) The effect of DHEA or G6PD knockdown on intracellular ATP:ADP ratio measured by PercevalHR fluorescence. Unpaired t-test. **p $<0.01$. (i-l) Working models for AMPK activation and NADPH level regulation in glucose-fed cells under oxidative stress. (a-h) All experiments were performed in glucose-fed HeLa cells. For panel Fig. 5c, f, " $\mathrm{R}$ " represents ratio of iNap1 corrected by iNapc. Unless otherwise indicated, data were represented as mean \pm s.e.m. 
a

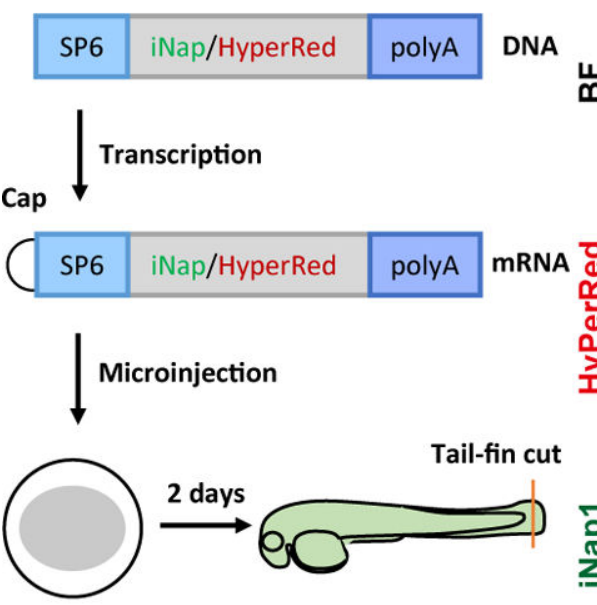

b
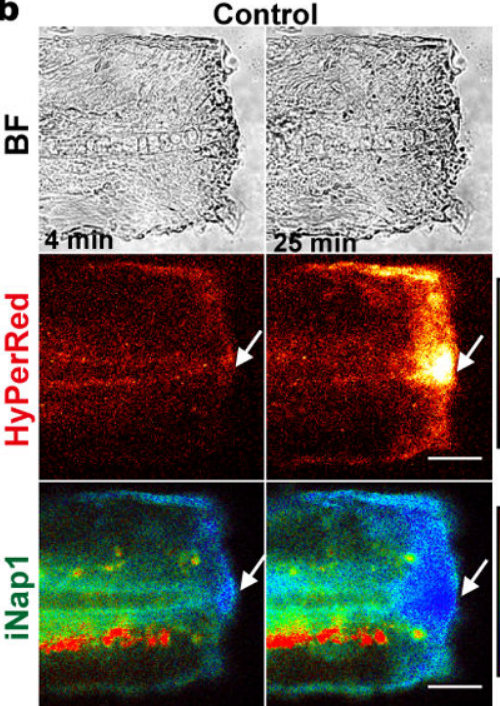

rol

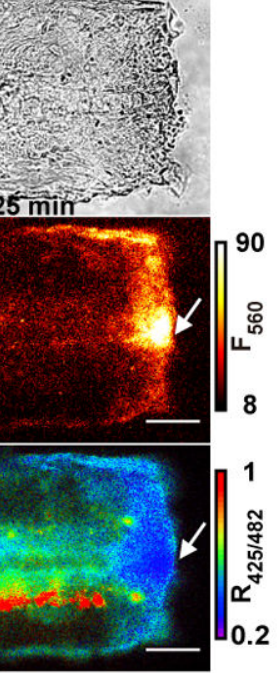

$100 \mu \mathrm{M} D P I$

㭊

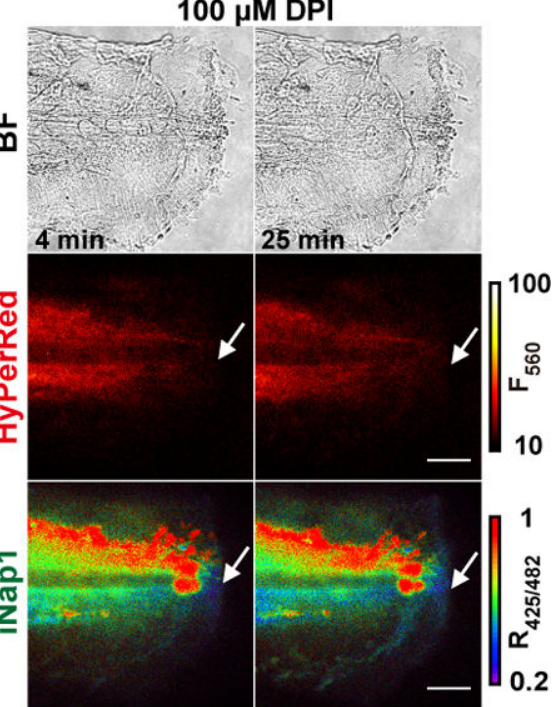

Figure 6. Simultaneous visualization of NADPH and $\mathrm{H}_{2} \mathrm{O}_{2}$ dynamics in wound margin by coexpression of iNap1 and HyPerRed in zebrafish larvae

(a) General transgenic zebrafish procedure. (b) Simultaneous visualization of NADPH and $\mathrm{H}_{2} \mathrm{O}_{2}$ dynamics in wound margin by co-expression of iNap1 and HyPerRed in zebrafish larvae with or without $100 \mu \mathrm{M}$ DPI. Tail-fin tip amputation was performed at the 0-min time point. Scale bar, $50 \mu \mathrm{m}$. 